\title{
Distributed Optical Fiber Sensing Based on Rayleigh Scattering
}

\author{
Luca Palmieri ${ }^{1, *}$ and Luca Schenato ${ }^{2}$
}

\author{
${ }^{I}$ Department of Information Engineering, University of Padova, 35131 Padova, Italy \\ ${ }^{2}$ Research Institute for Geo-Hydrological Protection, National Research Council of Italy, 35127 Padova, Italy
}

\begin{abstract}
Optical fiber sensors offer unprecedented features, the most unique of which is the ability of monitoring variations of the observed physical field with spatial continuity along the fiber. These distributed optical fiber sensors are based on the scattering processes that originate from the interaction between light and matter. Among the three different scattering processes that may take place in a fiber-namely Rayleigh, Raman and Brillouin scattering, this paper focuses on Rayleigh-based distributed optical fiber sensors. For a given optical frequency, Rayleigh-based sensors exploit the three main properties of light: intensity, phase and polarization. All these sensing mechanisms are reviewed, along with basic principles, main acquisition techniques and fields of application. Emphasis, however, will be put on polarization-based distributed optical fiber sensors. While they currently represent a niche, they offer promising unique features worth being considered in greater detail.
\end{abstract}

Keywords: Optical fiber sensor, distributed sensors, Rayleigh scattering, polarization.

\section{INTRODUCTION}

The idea of using an optical fiber as a sensing element is almost as old as the idea of using the fiber as a transmission medium [1]. As already highlighted in many review papers [2-11] and testified by a vast literature, optical fiber sensors (OFS) offer many advantages with respect to electrical and mechanical ones. What is most attracting is their being passive, little intrusive and intrinsically immune to electromagnetic interference, as well as the relative cheapness of the sensing element and the easiness of multiplexing, qualities that make them particularly be fitted to environments where harshness, geographical extension or required density of sensing points is demanding [12-14].

The very unique property of OFSs, unparalleled by any other technique, is the ability of monitoring physical fields (such as temperature, strain, magnetic field, chemicals concentration, etc.) distributedly along the fiber. These distributed OFSs (DOFS) map physical fields acting on the fiber by exploiting the scattering processes that take place in it, and probing the fiber with proper interrogation systems. Two parameters are distinctive of DOFSs: measurement range and spatial resolution. Measurement range simply represents the maximum length of the sensing fiber and may vary from less than one meter to more than $100 \mathrm{~km}$, depending on the monitored physical field and on the sensing method actually employed. Spatial resolution represents, loosely speaking, the spatial accuracy with which the physical field can be mapped, and may range from millimeters to some meters or above. A more precise definition of this parameter requires,

*Address correspondence to this author at the Department of Information Engineering, University of Padova, 35131 Padova, Italy;

Tel: +39049 827 7785; Fax: +39049 827 7699;

E-mail: luca.palmieri@unipd.it however, subtle considerations that are deferred to the next sections. Of course, a compromise between measurement range and spatial resolution must be accepted, for in general a sharper spatial resolution requires a probe light with wider bandwidth, which is more difficult to detect and to propagate without distortion over a long distance. This compromise is usually quantified by the ratio between measurement range and spatial resolution, which can be interpreted as the number of equivalent point sensors offered by a DOFS. This figure may be as high as some tens of thousands, and it is one of the reasons why DOFSs can be more advantageous than point sensors, especially when the area to be monitored is extended and/or when the required density of sensing points is high.

Three different scattering processes may take place in an optical fiber and can be exploited in a DOFS, namely: Raman, Brillouin and Rayleigh scattering [15]. When the fiber is probed with an optical signal, each of these scattering processes may generate a back propagating light than can be used to "read" the local properties of the fiber and hence to infer information on the surrounding environment. There is however a deep difference between Raman and Brillouin scattering, on one side, and Rayleigh scattering, on the other. In fact, the intensity of the (anti-Stokes) Raman scattering has an intrinsic dependence on the temperature of the fiber, which has been exploited to implement very successful distributed temperature sensors (DTS). Similarly, the frequency of Brillouin scattering is intrinsically dependent on fiber density, which in its turn depends intrinsically on temperature and strain, a feature that is largely used in Brillouinbased DOFSs [12-14]. On the contrary, Rayleigh scattering in optical fiber is per se independent of almost any external physical field for a wide range of conditions $[15,16]$. Actually, in Rayleigh-based DOFSs the scattering itself is used only to track and to reveal propagation effects (in the most 


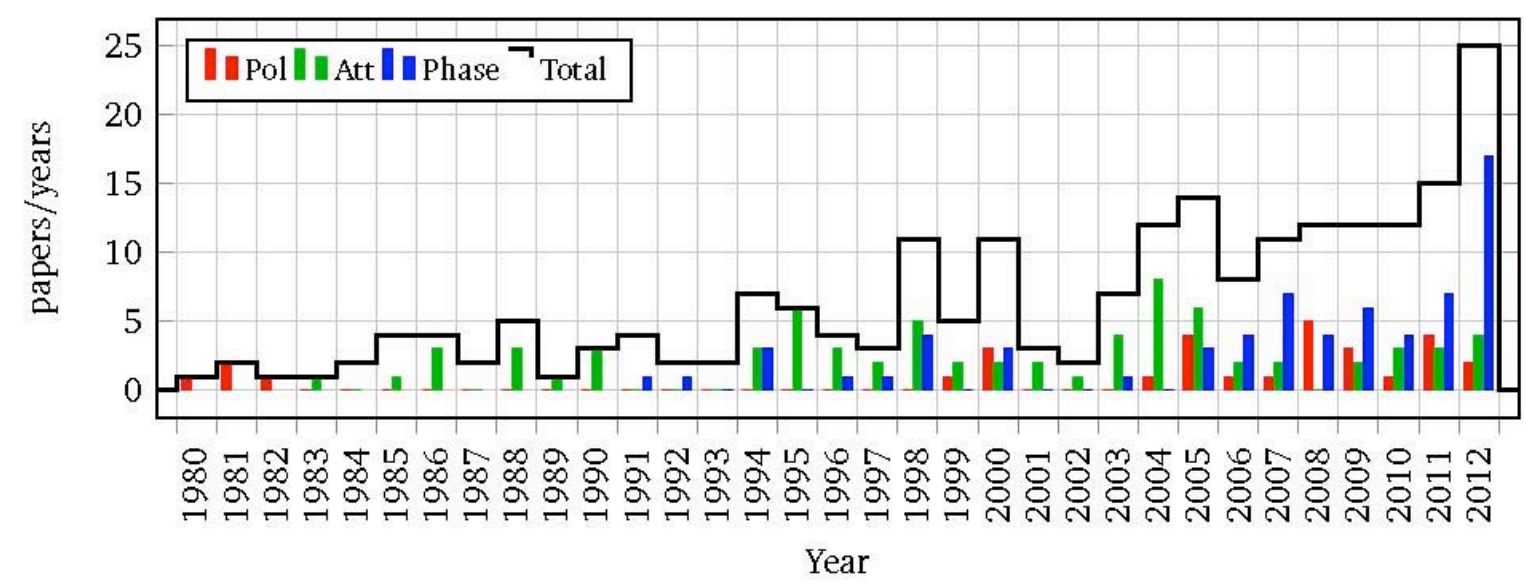

Fig. (1). Number of papers published per year about Rayleigh-based DOFSs in the main international peer-review scientific journals and conference proceedings. Red, green and blue beams refer to polarization, attenuation, and phase interference based DOFSs, respectively. Solid line represents the total number of papers per year, including review and general ones.

wide sense), which are the real sensing mechanisms. These propagation effects include attenuation and gain, phase interference and polarization variation, and all of them can be exploited to implement a Rayleigh-based DOFS. It's worthwhile remarking that these propagation effects may affect also Raman- and Brillouin-based DOFS, but they are usually neglected just because Raman and Brillouin scattering offer direct sensing mechanisms.

Because of this peculiar characteristic, Rayleigh-based DOFSs are potentially sensitive to many different physical fields, beside temperature and strain. For example, attenuation induced by coupling to radiation modes (e.g. microbending effects or evanescent field interactions) has been exploited to sense parameters as diverse as relative humidity [17-25], concentration of chemicals [26-47], strain [48-58] and pressure [26, 59]. Radiation-induced fiber darkening is currently investigated as transducing mechanism for Rayleigh-based DOFS to monitor ionizing radiations [6062]. Phase interference is at the ground of Rayleigh-based vibration and intrusion DOFS [63-84], and is the physical phenomenon behind the so called "fiber fingerprint", the paradigm of a successful commercial implementation of Rayleigh-based DOFS for strain and temperature monitoring [85]. Finally polarization of light plays a very unique role in Rayleigh-based DOFS, enabling the sensing of quantities like magnetic field, twist and geometrical layout.

In the light of their potential versatility, this paper reviews the current state of art of Rayleigh-based DOFSs, addressing acquisition techniques, sensing mechanisms and applications. Given their unique features, and since they are relatively less known, most emphasis is given to Rayleighbased DOFSs that exploit polarization variation as sensing mechanism.

\subsection{Guide to the Reading}

As shown in Fig. (1) the literature about Rayleigh-based DOFS is rather wide and research activity in this field started more than 30 years ago. Among the different stand points that can be chosen to explore this topic, we have selected two of them: acquisition techniques and sensing mechanisms.
The first part of the paper, namely Sec.2, is dedicated to a review of the acquisition techniques that can be used to detect Rayleigh back scattering. Time and frequency domain approaches will be described separately, discussing their respective principles, weakness and strengths. This section is didactic to some extent, but we believe that the fundamental principles at the base of DOFS deserve special attention.

Subsequently, the sensing mechanisms exploited in Rayleigh-based DOFSs are reviewed. We have grouped these mechanisms in three main categories: attenuation, phase interference and polarization variation.

Section 3 is devoted to sensors based on attenuation, which includes macro and micro-bending, coupling to radiation modes and, marginally, absorption and amplification. As shown in Fig. (1) attenuation is the sensing mechanism that has been investigated with more continuity over the years, likely because of its relative simplicity. Differently, DOFSs based on phase interference started receiving great attention only in the recent years, and are one of the reasons of the overall increasing interest in Rayleigh-based DOFSs. This class of DOFSs is reviewed in Sec.4, whereas Sec.5 is dedicated to polarization based DOFSs. Fig. (1) shows that polarization variation has been the first sensing mechanism to be explored [86], but only recently it started attracting a more constant attention. As anticipated above, most of this review is focused on these polarization based DOFSs, which are analyzed in greater detail. Overall, more than 200 papers on Rayleigh-based DOFSs have been published on international journals and conference proceedings, to date; general comments about them are given throughout the text, while a schematic classification by field of application in given in Sec.6, along with some concluding remarks.

In this review we restrict the analysis to DOFSs where Rayleigh scattering plays a key role. Therefore, techniques where Rayleigh scattering is used as a reference for other scattering processes are not considered. Similarly, we do not discuss neither approaches where reflectometric techniques are used only to interrogate concatenations of partially reflective devices (such as fiber Bragg gratings), nor techniques based on the joint analysis of transmitted and reflected light. Finally, hereinafter by "DOFS" we mean dis- 
tributed sensors based on Rayleigh scattering, unless differently specified.

\section{ACQUISITION METHODS}

As recalled in the Introduction, Rayleigh-based DOFSs exploit scattering to track and reveal propagation effects. Different theoretical models have been proposed to describe Rayleigh scattering [87-94]. The interested reader is referred to the literature for details; here, we recall only some basic concepts for completeness.

Rayleigh scattering is an elastic scattering process that originates from density fluctuation in the material $[14,93$, 94]. When the scattering takes place in an optical fiber, part of the scattered light (which in general radiates in all directions) is captured by the guiding structure of the fiber itself and back propagates to the fiber input. This "backscattered light" is the signal detected by Rayleigh-based DOFSs; it is in general rather weak (several tens of dB less than the incident light), and this fact represents the main technical difficulty in Rayleigh-base DOFSs.

From a phenomenological point of view, the backscattered light may be described as the coherent super position of the light generated by many weak reflectors, randomly spread in the fiber [95, 96]. Each of these reflectors can be described by means of Rayleigh's theory [94], according to which the backscattered light is in phase with the incident one and has the same polarization (the subtle details of this last claim are discussed in Sec.5). Differently, the intensity of the light reflected by the single scattering center is a random quantity, because so are density fluctuations. As a consequence, neglecting for simplicity dispersion and polarization effects, the complex envelope, $b(t)$, of the backscattered light in a single mode fiber can be qualitatively described by the following expression [97] :

$b(t)=\sum_{n} c_{n} \mathrm{e}^{-2\left[\alpha\left(z_{n}\right)+j \beta z_{n}\right]} a\left(t-2 \tau_{n}\right)$,

where $\beta$ is the propagation constant of the fiber, $\alpha(z)$ describes the attenuation accumulated up to $z, c n$ and $z n$ are the random amplitude and position of the $n$th scattering center, $\tau_{n}=z_{n d} \beta / d \omega$ is the group delay introduced by the propagation up to $z_{n}$, the factor 2 takes in to account roundtrip propagation, and $a(t)$ is the complex envelope of the signal used to probe the fiber - note that this is in general an arbitrary wave function. In this context the exact statistics of $c_{n}$ and $z_{n}$ is not important. Differently, it is worth while remarking that these numbers can be considered constant as long as the fiber is not perturbed.

In its simplest configuration, the aim of the acquisition techniques is to retrieve the attenuation $\alpha(z)$ by measuring $b(t)$. Basically, this is equivalent to measuring the response of the "transmission" channel made by roundtrip propagation through the fiber. Just as for general channels, also this equivalent roundtrip channel can be characterized either in the time domain, by probing the fiber with ideal pulses to measure its impulse response, or in the frequency domain, by probing the fiber with continuous wave $(\mathrm{CW})$ signals at different frequencies to measure its frequency response. The

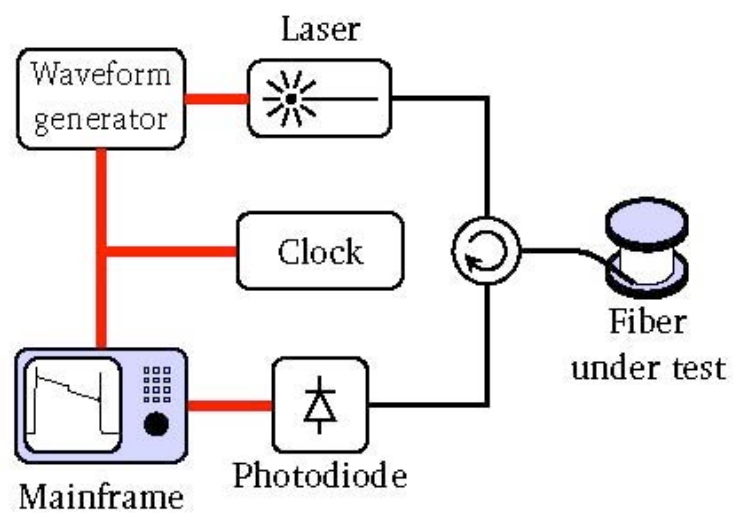

Fig. (2). Schematic setup of a generic optical reflectometer.

two approaches lead to two different classes of reflectometers.

\subsection{Time Domain}

The optical time domain reflectometer (OTDR) has been proposed for the first time by Barnoski and Jensen in 1976 [98]. Its working principle is relatively simple, and consists in sending a single pulse in the fiber to measure the impulse response of roundtrip propagation. Correspondingly, also the implementation is in principle simple (see Fig. 2), and basically includes a source of optical pulses, a photo-receiver, proper optical circuitry (such as couplers or circulators) to separate forward path from the backward one, and proper electronics to control the setup and to process and store recorded data.

Of course, the shorter the pulse, the more accurate the measurement of the impulse response. One way to understand the consequence of using non ideal pulses is resorting to a Minkowski diagram (see Fig. 3). The abscissa represents time, whereas the ordinate represents space normalized to group velocity. For simplicity we consider a rectangular pulse, and we assume that at time $t=0$ it is about to enter the fiber, therefore in the diagram it occupies the space represented by the red segment $O T$. As the pulse forward propagates in the fiber, its leading edge traces in the diagram the half line $\mathcal{L}$ and, almost continuously, it gives rise to backscattered light. For simplicity, let us consider just the scattering that takes place in point $A$, i.e. at time $t_{A}$. Backscattered light originated from there back propagates tracing the segment $A C$, and reaches the fiber in put at time $2 t_{A}$. Meanwhile, also the rest of the pulse propagates in the fiber. The pulse trailing edge, in particular, traces the half line $\mathcal{T}^{\mathcal{T}}$ and contributes to the back scattering as well. Among all the scattering contributions, let's focus on that originated in point $B$. With respect to the scattering in $A$, the scattering in $B$ takes place later in time but before in space, in such a way that the two contributions arrive overlapped at the fiber input at time $2 t_{A}$.

We evince from the diagram that, actually, all the scattering contributions that take place in the space time region described by segment $A B$ arrive overlapped at the fiber in put at time $2 t_{A}$. Spoken in other way, the power measured by the OTDR receiver at time $2 t_{A}$ is the super position of the scattering generated from segment $A B$. Simple geometrical considerations lead to conclude that this corresponds to scatter- 


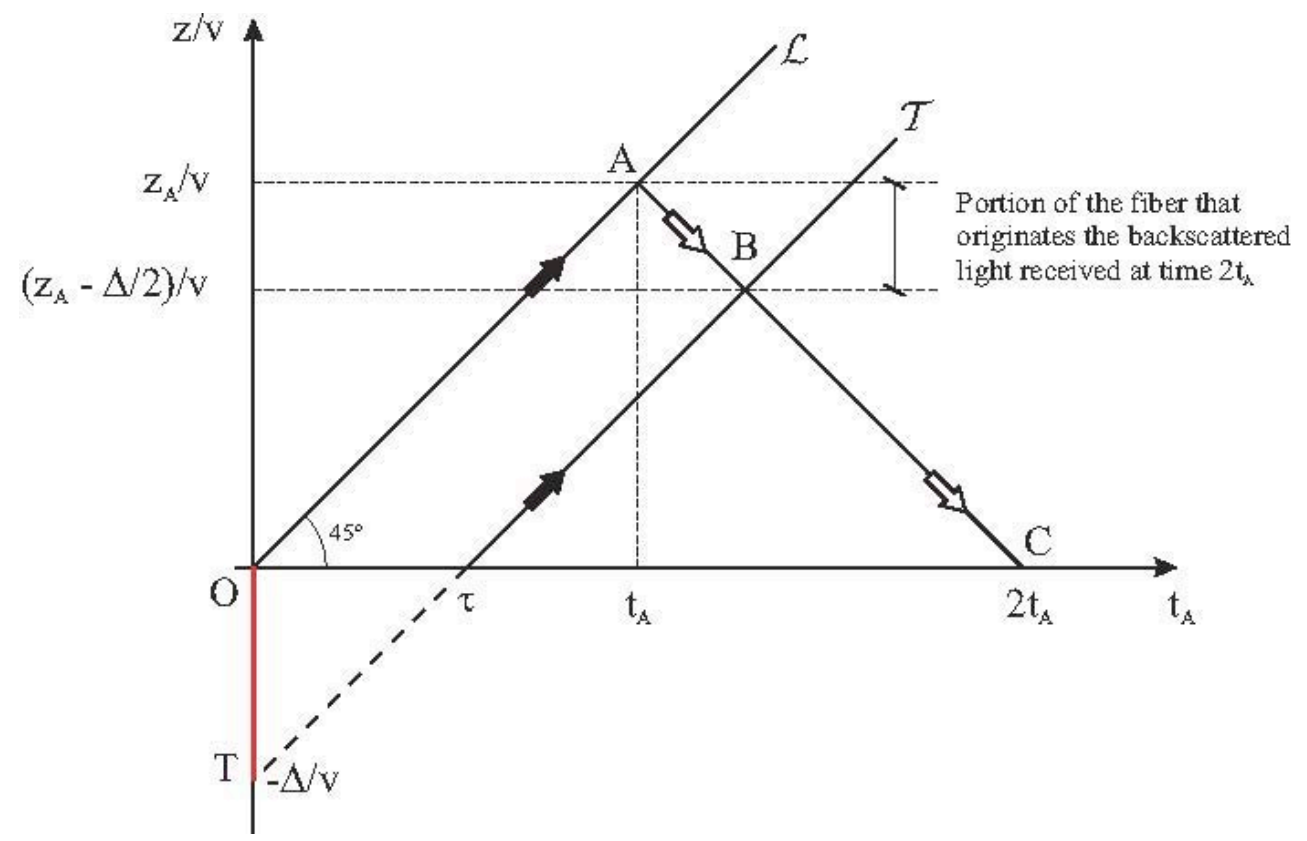

Fig. (3). Minkowski diagram sketching the propagation of a probe pulse and of the corresponding Rayleigh scattering.

ing generated by a section of fiber with length equal to half the pulse length.

The main consequence of the above argumentation is that the spatial resolution of an OTDR is equal to half the length of the probing pulse. Note, however, that this should be considered as a lower bound to the resolution. In fact, the resolution of a DOFS, i.e. the spatial accuracy with which the specific physical field is mapped, depends on other factors, such as the signal to noise ratio (SNR) of the measurement, for example. As a consequence, the spatial resolution of DOFSs is sometimes defined, with respect to a step variation of the monitored physical field, as the length of the sensor's transient response from $10 \%$ to $90 \%$ of the step nominal amplitude [99].

In order to increase the spatial resolution one should reduce the pulse length, but this causes a reduction of the SNR. Actually, the reasoning on the Minkowski diagram shows that the power backscattered by the fiber is not proportional to the pulse power, but rather to the pulse energy. For a given pulse power, halving the pulse duration, $\tau$, halves the backscattered power. Furthermore, the electrical bandwidth of the receiver must fit to the pulse duration; thus halving $\tau$ requires the receiver bandwidth to be doubled, and hence doubles the power of the noise affecting the measurement. As a result, the SNR is roughly proportional to the square of the spatial resolution. Halving the spatial resolution causes a four-fold reduction of the SNR, and this cannot be easily compensated by increasing the pulse power, because that would soon originate distorting nonlinear effects. One way to overcome this impasse is to use probe signals other than a single pulse. This idea is exploited by correlation-OTDR [100-102], for example, but the best performances are obtained by coded-OTDR (C-OTDR) [103-105].

A C-OTDR probes the fiber with a sequence of pulses encoded according to binary words of length $L$, chosen from an alphabet of $L$ linearly independent words. Assuming for example $L=3$, the three code words can be $w_{1}=(1,1,0)$, $w_{2}=(1,0,1)$, and $w_{3}=(0,1,1)$. Since $w_{1}+w_{2}-w_{3}=(2,0,0)$, we see that a proper linear combination of the OTDR traces recorded for each of the words, gives the trace that would have been obtained by launching a single pulse. As a result, the spatial resolution is preserved and, at the same time, the SNR of each measurement has been increased, since more than one pulse was sent in the fiber at the same time. Generalizing this concept to words of arbitrary length, it is possible to show that the code increase the SNR by a factor $(L+1) /\left(2 L^{1 / 2}\right)$, known as coding gain [103]. This extra SNR can then be used to increase measurement range, reduce measurement time, or improve spatial resolution. Note, however, that in this last case an increase in the receiver bandwidth (and consequent noise) is still required. As a consequence, halving the spatial resolution approximately requires a 64 fold increase in the code length.

Another way to improve spatial resolution is represented by the photon counting OTDR, $v$-OTDR [106, 107]. In this case, very short optical pulses are sent in the fiber, and the very low backscattered power is measured by an APD operated in Geiger mode. The APD is activated only for a time lapse $\Delta t$, with a delay $t_{D}$ from the pulse launch. Photons received in the time interval $\left[t_{D}, t_{D}+\Delta t\right]$ are counted for several pulses, and the resulting number represents the probability of receiving backscattered power from the specific portion of fiber, framed by the selected time interval. The complete map is obtained by scanning $t_{D}$. The advantage of $v$-OTDR is that the resolution is controlled by $\Delta t$ (as long as the optical pulse is shorter), and therefore spatial resolutions in the order of some centimeters can be obtained [108]. The drawback is the extremely long time of acquisition requested to count a statistically meaningful number of photons for each delay $t_{D}$.

As described in (1), the power receive data given time is the coherent upper position of many contributions from different scattering centers. Consequently, the received signal is affected by speckle noise, also known as Rayleigh fading noise $[109,110]$. To mitigate this source of noise, the tech- 


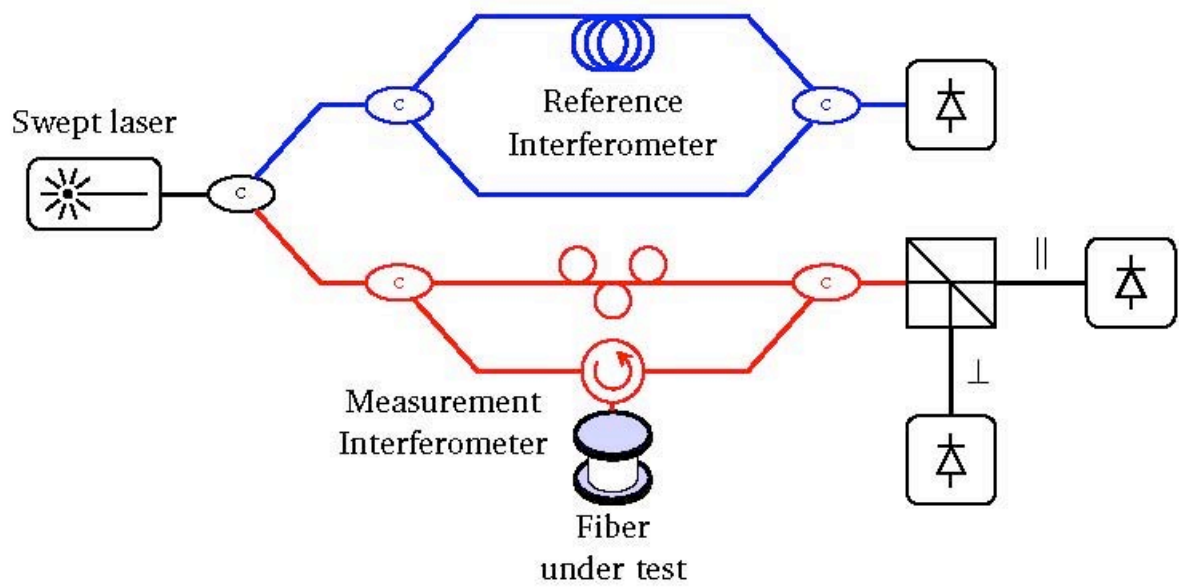

Fig. (4). Schematic of a possible OFDR implementation. Alternatively, the output of the reference interferometer can be used to a synchronously sample the output of the measurement interferometer to compensate frequency swept non linearity.

niques described above use low coherence (i.e. wide bandwidth) optical pulses, so to minimize coherent interference. On the other hand, however, these fringes of interference are related to the position of the scattering centers. Therefore, if the fiber undergoes a strain or a temperature variation, the relative position of the scattering centers changes, inducing a variation in the fringe pattern. This is exactly the basic principle of phase-OTDR ( $\phi$-OTDR) $[109,111,112]$. This kind of OTDR employ narrow-band, highly coherent optical pulses to enhance the interference fringes. As we will see, the typical application is sensing of vibration. $\phi$-OTDR suffers the same constraint on spatial resolution and measurement range as standard OTDR. In particular, spatial resolution is usually in the order of meters.

\subsection{Frequency Domain}

Frequency domain analysis is the dual way to characterize the equivalent roundtrip channel, and consists in measuring its frequency response. Two different approaches can be exploited: incoherent optical time domain reflectometry (IOFDR) and coherent optical time domain reflectometry (OFDR) also known as frequency modulated continuous wave (FMCW) technique.

The setup of an I-OFDR is basically the same shown in Fig. (2). The main difference is that the source is not pulsed, but generates $\mathrm{CW}$ light modulated in amplitude by an RF signal. The RF frequency is linearly swept to probe the frequency response in a given bandwidth. Similarly to the time domain, the spatial resolution is still inversely proportional to the source bandwidth, i.e. to the bandwidth swept by the RF modulation. Therefore, a wide bandwidth receiver is still needed to achieve high spatial resolution. However, owing to the RF modulation, I-OFDR may take advantage of electrical heterodyne receivers and their enhanced sensitivity. As a result, I-OFDR may offer better performances with respect to OTDR, at least in certain cases $[23,56]$.

The best performances in term of spatial resolution and sensitivity are however achieved by OFDR [85, 113, 114]. The schematic of an OFDR is shown in Fig. (4). The source is a highly coherent $\mathrm{CW}$ laser, whose optical frequency is linearly swept in a bandwidth that may extend to several tens of nanometers. Part of this probe signal is sent in the fiber under test, and part is used as a local oscillator (LO). The backscattered light is then mixed with this LO and the resulting coherent interference is detected by the receiver. This solution offers two main advantages. The mixing with the LO is basically a sort of very effective "intermediate frequency" demodulation; actually, by properly tuning the swept speed, the tens of nanometers bandwidth scanned by the source is "squeezed" to a much more manageable electric signal with a bandwidth of few MHz. At the same time, heterodyne reception allows to achieve extremely high sensitivity. The spatial resolution of an OFDR is $\lambda_{s} \lambda_{f} /(2 n \Delta \lambda)$, where $\lambda_{s}$ and $\lambda_{f}$ are the lower and upper wave lengths of the frequency scan, $\Delta \lambda=\lambda_{f}-\lambda_{s}$, and $n$ is the effective refractive index of the fiber. As a result, by scanning some tens of nanometers, OFDR can nominally achieve sub millimeter spatial resolution.

Of course, all these advantages come at the cost of an increased complexity and limited range of measurement. Actually, performances may seriously degrade if the optical frequency is not linearly swept; for this reason proper solution must be implemented to compensate possible non linearity. As an example, the setup shown in Fig. (4) exploits a MachZehnder interferometer to measure the actual optical frequency. To get the best out of heterodyne reception, balanced photodiodes must be used. Furthermore, being based on optical interference, OFDR is intrinsically sensitive to polarization; therefore a polarization diversity receiver must be used. The most limiting factor in the OFDR is however the source coherence length. Clearly, for the principle to work, there must be coherence between the LO and the backscattered light. As a result, the measurement range of the OFDR is limited by the coherence length of the source. Submillimeter spatial resolution is therefore achieved only over fiber lengths in the order of few tens of meters. Few kilometers of range have been reported, but in this case the spatial resolution is the order of several millimeters. It is worth while mentioning a recently proposed OFDR setup, in which the measurement range has been extended to few tens of kilometers, by properly compensating the phase variation due to moderate lack of coherence $[115,116]$. 


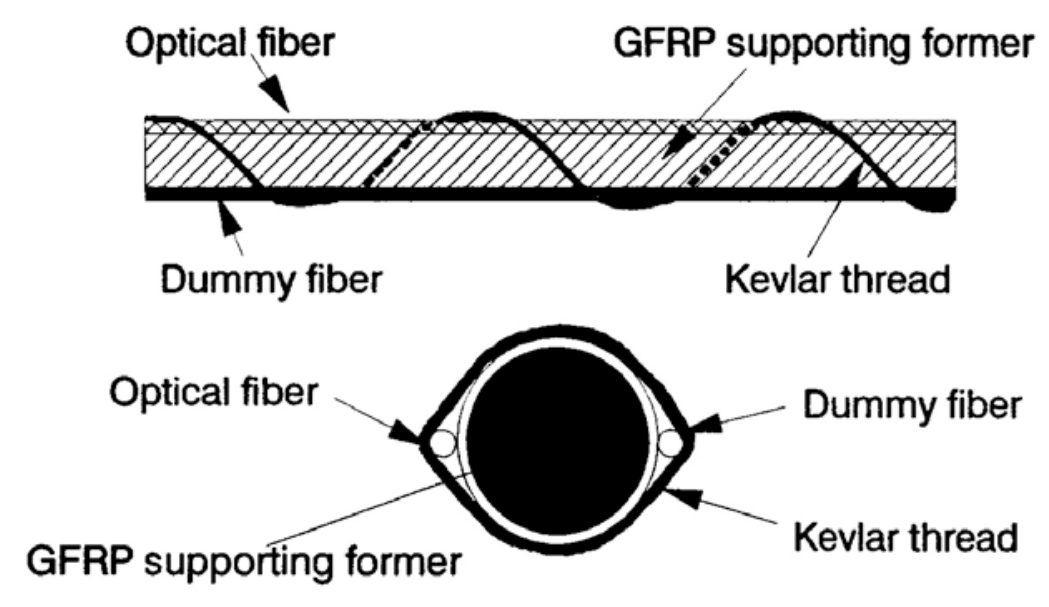

\section{Cross section}

Fig. (5). Example of micro-bending sensors based on swellable polymer. When the polymer enters in contact with a proper liquid (either water, solvent or hydrocarbon), the polymer swells pressing the fiber against the thread, which induces micro-bending loss. Reprinted with permission from Ref. [28].

\section{DOFS BASED ON ATTENUATION}

DOFS based on the measurement of fiber attenuation are intrinsically sensitive to the following effects:

1. coupling of guided modes to radiation modes (or leaky modes, as some times they are loosely called [117]), which can be more finely sub divided in:

(a) micro- and macro-bending;

(b) interaction through evanescent fields (i.e. cladding "tails");

(c) mode coupling in multi modal polymer optical fibers (POF).

2. absorption (or gain) of the material.

These effects are used in different ways to make the fiber sensitive to several physical parameters, ranging from temperature to chemicals concentration as reviewed in the following. In most of the cases, attenuation is measured by means of OTDR or I-OFDR, where as OFDR finds marginal application.

\subsection{Micro- and Macro-Bending}

Sensors based on bending effects are usually considered "extrinsic sensors", because the sensing mechanism is not intrinsic to the fiber, but has to be mechanically induced by an external structure.

The most common solution are based on micro-bending, which is induced on the fiber by a proper periodic structure applied around it $[19-24,26,28,29,34,37,38,48,50-52$, 57, 118-124]. The first proposal of this kind we are aware of dates back to 1989 , and consisted in a periodic metallic coating applied to a fiber, to make it sensitive to pressure, temperature and molecular hydrogen [26]. However, more convincing and viable configurations are those based on swell able polymer materials [19, 20, 28, 29, 34, 37, 38]. For example, Michie et al. [28, 29] proposed the structure shown in Fig. (5), where a multimodal fiber was constrained to a water swell able support by a helically wound Kevlar thread. Whenever the structure comes in contact with water, the supports wells pressing the fiber against the Kevlar thread, hence inducing micro-bending loss, which can be detected by a simple OTDR setup. A very similar approach has been proposed by MacLean et al. for the detection of hydrocarbon and solvent spills [34, 37, 38], and by Pierce et al. for relative humidity monitoring [23, 120].

Other fields of application of micro- and macro-bending are crack detection [50,118, 124], strain and deformation sensing [48], and land slide monitoring [51, 52, 57]. In these cases special lumped structures exert periodic perturbations on relatively short sections of fiber, where the micro- or macro-bending is induced. As a result these sensors are quasi-distributed.

\subsection{Evanescent Field}

Sensors based on evanescent field interaction, are those where the cladding of the fiber is modified or properly engineered to enhance the interaction between the propagating light and the external environment [18, 25, 27, 30-33, 35, 36, $39-47,125,126]$. This interaction results in an increased attenuation or and increased Rayleigh scattering, which can both be measured by standard OTDR. In general, DOFSs of this kind exploit multimodal fibers, taking advantage of the thinner cladding. The use of such fibers, however, limits the measurement range to about $1 \mathrm{~km}$.

If we exclude a few early examples of temperature monitoring $[125,126]$, the main field of application of this kind of sensors is chemicals detection. Two main approaches are usually adopted. In one approach, the fiber cladding is made with a proper material that changes its optical properties (either absorption or refractive index) when in contact with the target analyte. In a second approach, the cladding is almost completely removed in short fiber sections, which therefore become sensitive to the refractive index of the medium surrounding the section. Of course, this last solution leads to quasi-distributed sensors. 


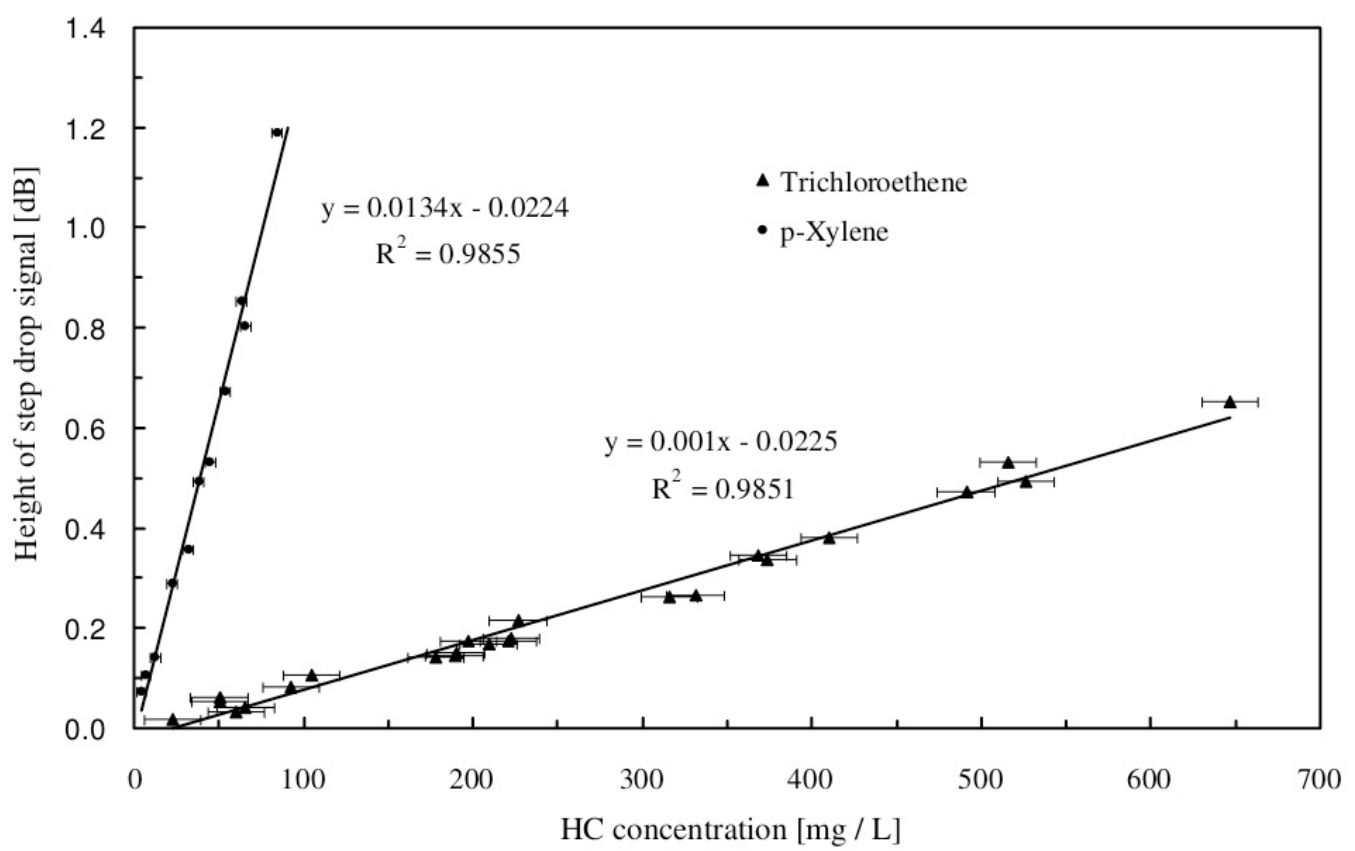

Fig. (6). Loss (as measured with an OTDR) induced on a 2-m-long section of fiber with silicone cladding, as a consequence of contact with aqueous solutions of $p$-xylene and trichloroethene. Values are normalized to the loss given by exposure to pure water. Reprinted with permission from Ref. [35].

A nearly example of the first approach is the use of polyvinyl acetate cladding to detect humidity and water [17]. More recently, different cladding have been proposed to induce sensitivity to other chemicals, such as hydro-carbons [35], hydrogen cyanide [41], chlorine [39, 42], and hydrogen sulfide [42]. The sensing mechanism is either a change of cladding absorption or an increase of cladding refractive index, so to compromise fiber guidance. This is for example the approach exploited by Buerck et al., where the refractive index variation induced by various hydrocarbons, both pure or dissolved in water, on a silicone polymer cladding are extensively analyzed [35]. The work shows how hydrocarbons dissolved in water can be detected using an OTDRbased configuration, with a spatial resolution of about 2 mover distance of about $1 \mathrm{~km}$, for concentrations ranging from few $\mathrm{mg} / \mathrm{l}$ to few hundreds of $\mathrm{mg} / \mathrm{l}$, depending on the hydrocarbon (see Fig. 6).

The evanescent field interaction is exploited also by quasi-distributed sensors, where "windows" are opened along the fiber cladding to enable interaction of the propagating light with the analyte. Yun et al., for example, recently proposed a quasi-distributed sensor for various chemical elements, made by optically ablating part of the cladding from a graded index POF [47]. A simpler approach is proposed by Kinet et al., which create sensing points along the fiber by damaging the guiding structure of the fiber using a splicer and a well calibrated discharge arc [45]. In these spots light tends to diffuse more in the cladding where it can interact with the analyte surrounding the fiber. In particular, the lower the refractive index of the analyte, the larger the local peak of reflection, which is measured with a $v$-OTDR.

\subsection{Mode Coupling in Multimodal POF}

Despite strictly speaking bending effects and evanescent field interactions described above are all mode coupling ef- fects, the mode coupling that may take place in a multimodal POF deserves a separate classification. Actually, POFs may tolerate much larger strain and stress than silica fibers, to the extent that very high mode coupling can be induced in them. A survey of this effect is given by Husdi et al. [127]. They show, for example, that by twisting a $1 \mathrm{~cm}$ long section of POF by more than 1 turn, a localized reflection is induced whose amplitude is roughly linearly related to the twist rate.

In the recent years a few papers have exploited the idea of using a bare POF as a strain sensor based on this mechanism $[25,53,55,56]$. Preliminary results showed that strain as high as $100 \%$ can be measured over distances of about half a kilometer [55], suggesting that POF-based strain sensors might be a promising and viable solution for structural health monitoring (SHM).

\subsection{Absorption and Gain}

There are few examples of DOFS based on absorption and gain [60-62, 128-130]. Among them is the first DOFS based on OTDR analysis and, probably, the first example of distributed temperature sensor, which dates back to 1983 , and was based on the temperature dependence of the Rayleigh scattering in a liquid core fiber [128]. Other solutions were based on the dependence on temperature of absorption in niobium-doped fibers [129], and of gain inerbium-doped fibers [130]. These ideas, however, have not received much attention.

On the contrary, there is a certain interest around the idea of using the fiber as a sensor of ionizing radiation, exploiting the darkening effect that fibers undergo when exposed to such radiation [60-62]. The sensitivity of this effect may vary substantially from fiber to fiber, depending on dopants and geometry. Rare Heart doped fiber can have sensitivity of few tenths of $\mathrm{dB} / \mathrm{mGy}$ at $830 \mathrm{~nm}$ and about one order of 


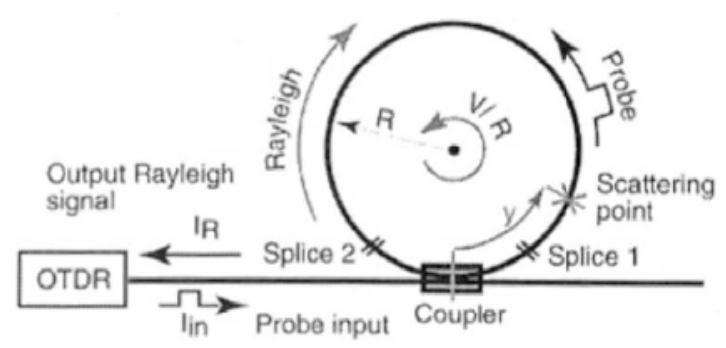

(a)

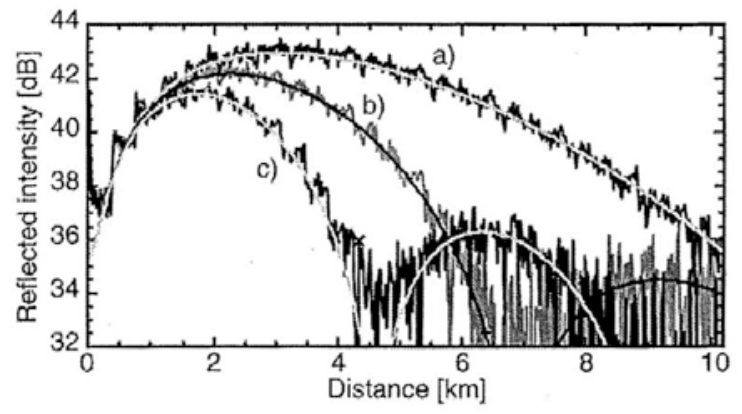

(b)

Fig. (7). Experimental setup of the Rayleigh-based fiber gyroscope. Reprinted with permission from Ref. [169].

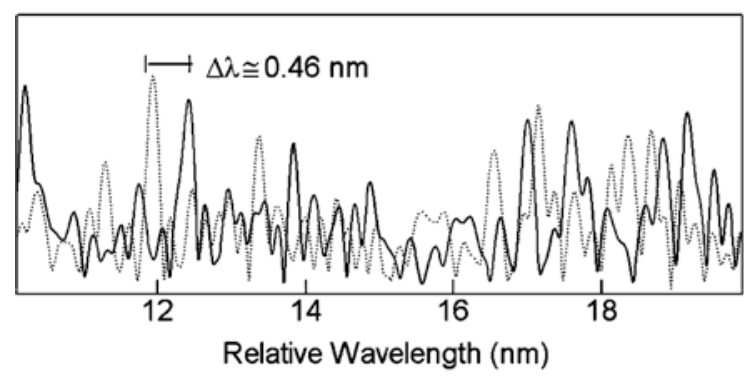

(a)

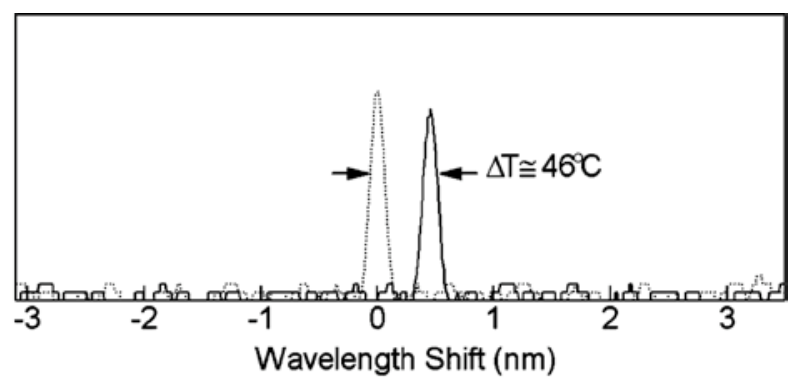

(b)

Fig. (8). Spectral shift induced by thermal variation in an equivalent FBG made by a $5 \mathrm{~mm}$ long section of fiber. (a) Spectral response of the fiber section before (dashed) and after (solid) heating. (b) Auto-correlation of the spectral response before heating (dashed) and crosscorrelation between the two spectra after heating (solid). Reprinted with permission from Ref. [140].

magnitude less at $1310 \mathrm{~nm}$ [61]. Similar sensitivity can be achieved by properly selecting commercial germaniumdoped, phosphorous co-doped, telecommunication fibers, which represent a much cheaper alternative [62]. Research activity in this field is not very active; nonetheless, this kind of radiation DOFSs has interesting applications in fields ranging from medical dosimetry, to monitoring of nuclear waste disposal sites and of particle accelerators.

\section{DOFS BASED ON PHASE INTERFERENCE}

As described in Sec.2, owing to the "granularity" of the scattering centers, when the Rayleigh scattering is stimulated by a highly coherent light, the backscattered light is characterized by marked interference fringes, which change whenever the relative positions of the scattering centers vary. Consequently, DOFSs based on phase interference are intrinsically sensitive to temperature and strain variations, and the sensitivity is so effective that these systems are the only Rayleigh-based DOFSs successfully commercialized to date DOFSs based on phase interference may be divided in two classes, depending on whether the fiber is probed in frequency domain $[80,84,85,131-159]$ or time domain [63-73, $75,77,79,81,82,160-171]$. The physical effect is exactly the same for both, but the wide difference in spatial resolution and measurement speed of the two approaches leads to markedly different applications.

Before analyzing those two approaches in details, it is worthwhile remarking, for the sake of completeness, that there is a third minor class of Rayleigh-based sensors that exploit interference, namely: Rayleigh-based gyroscopes
[169]. These gyroscopes do not rely on the "granularity" of scattering centers, but on the Sagnac effect in fiber loops. Strictly speaking they are not distributed, yet they use a distributed measurement to measure the local rotation. The setup is shown in Fig. (7a) and consists in an OTDR connected to a fiber loop. Typical traces measured with that arrangement are shown in Fig. (7b) for different rotation speeds. The characteristic shape is a consequence of the pulse recirculating in the loop and of Sagnac effect. Simple as it is, this gyroscope has an accuracy of $0.01 \mathrm{rad} / \mathrm{s}$; however, it has received only sporadic interest $[170,171]$.

\subsection{Frequency Domain}

The idea of using an OFDR to accurately measure the fringe pattern of Rayleigh backscattered light was first proposed in 1992 by Juškaitis et al. [131], and subsequently applied to the measure of temperature [132]. A great impulse to this approach was given however in 1998 by Froggatt and Moore [85], who described the random fluctuation of the index of refraction that causes Rayleigh scattering as an equivalent random Bragg grating. According to this model, the fiber is (ideally) divided in short sections in the orders of centimeters, each of which is considered as a weak random fiber Bragg grating (FBG). It has to be stressed that, despite random, this equivalent weak FBG is stable in time, as long as the fiber is not perturbed. The frequency scan performed by the OFDR allows to retrieve the spectral response of each of this equivalent FBG. As in standard FBG analysis, the reflected spectrum shifts in response to temperature and strain variations (see Fig. 8). Therefore, by monitoring this shift (usually by means of correlation analysis) it is possible 
to measure the local change in temperature or strain. More specifically, the spectral shift can be expressed as $\Delta \lambda \simeq$ $K_{T} \Delta T+K_{\varepsilon} \varepsilon$, where, for standard G.652 Ge-doped silica fibers, the temperature coefficient $K_{T}$ is about $10 \mathrm{pm} /{ }^{\circ} \mathrm{C}$, and the strain coefficient $K_{\varepsilon}$ is about $1.2 \mu \mathrm{m} / \varepsilon$ [134, 140, 141]. Systems based on this approach may reach temperature and strain accuracy of about $0.1^{\circ} \mathrm{C}$ and $1 \mu \varepsilon$, respectively, over distances of few hundreds of meters and with spatial resolution around few centimeters. Note, however, that in general there might be an ambiguity in interpreting whether the spectral shift is due to temperature or strain variation, just as in FBG.

The effectiveness of this approach, along with the availability of a commercial device implementing it, has given rise to many works describing applications in various fields $[11,26,125,126,128-130,132,134,136,140,141,149$, $150,153,154,160,165-168,172-180]$. Temperature sensitivity, for example, has been tested down to $4 \mathrm{~K}[149,153]$, and has been exploited in a liquid level sensor, to be operated at cryogenic temperature [153]. The sensor is based on a metal coated fiber that is partly dept in the liquid and electrically heated. By measuring the distributed temperature along the fiber is then possible to understand which is the portion actually submerged, thus inferring the liquid level. The sensor has been successfully tested on liquid helium at $4 \mathrm{~K}$. A recent analysis has also suggested that this sensitivity to cryogenic temperature may be used in the monitoring of super conducting cables [149]. The viability of the technique to monitor temperature in a radiation environment such as those of a nuclear reactor has been verified, too [140].

Applications based on strain sensitivity are even more various. Chen et al. implemented a distributed hydrogen sensor by measuring the strain induced by hydrogen absorption along a palladium-coated fiber [151]. Sensitivity of few micro strain per percent of hydrogen concentration, with response time of few minutes, have been reported. SHM is another large field of application. Detailed experiments about monitoring of concrete structures have been reported by Villalba and Casas [158] and by Henault et al. [156]. In the frame work of a system to detect sinkholes and embedded soil cavities, Lanticq et al. compared an OFDR-based strain sensor with a Brillouin-based one [142]. Tests performed on a railway tunnel suggested that the OFDR-based system is more effective owing to its better spatial resolution.

Strain sensitivity has been used also to measure pressure and lateral strain. In general, this specific sensitivity is achieved by measuring the strain along the two polarization axes of a polarization maintaining (PM) fiber [143, 145, 152]. A differential analysis allows then to detect not only the magnitude of pressure, but also its direction with respect to the fiber birefringence axis. Maier et al., for example, have proposed a system in which the sensing PM fiber is terminated with a Faraday rotating mirror. In this way, both polarization axes are analyzed in a single frequency scan, without the need of any polarization switching at the fiber input [145]. An almost linear response up to load of $16 \mathrm{~N} / \mathrm{mm}$ has been reported. Chen et al. employed a similar approach to monitor pressure, by measuring the birefringence induced in a twin air hole photonic crystal fiber [152].
A last intriguing example of application is shape sensing $[135,137,157]$. In this case one or more fibers are properly arranged (either helically, or along the directrix) on a flexible tubular structure. The strain is measured along each of these fibers, so that, by means of a differential analysis, the path along which the tubular structure is laid can be calculated. Alternatively, the same goal can be reached by measuring the strain along the cores of a multi core fiber. Resolution in the order of $1 \%$ by length over fiber lengths of few tens of meter scan be achieved.

We conclude noting that the methods described above, being based on OFDR, are limited to relatively short measurement lengths. Some hundreds of meters have been reported, but the best performance is achieved in the range of few tens of meters. None the less, given the spatial resolution of few centimeters or less, this method provides a high density of sensing points over short distances, filling a range of application not easily covered neither by Brillouin nor by Raman based DOFSs.

\subsection{Time Domain}

The same idea exploited in OFDR measurements described above, can in principle be applied also in time domain, exploiting the phase interference created by the multiple scattering points within the probing pulse (see Fig. 3). In practice, this can be done by just using a $\phi$-OTDR [163]. Juškaitis et al. were the first to propose this solution in 1994, although their first experiments were still made with an OFDR [63].

The great advantage of this new approach is that it does not need a local oscillator to perform the measurement, because the interference fringes are build "within" the probing pulse. As a result, the measurement range of the system is no longer constrained by the coherence length of the fiber.

This simplification, however, comes at a cost: actually, $\phi$ OTDR usually has lower spatial resolution compared with OFDR and, consequently, the spectral response of the equivalent weak FBGs cannot be measured with an accuracy good enough to enable temperature or strain measurements. A noticeable exception is represented by the work of Koyamada et al. [165-167], where source and receiver of the $\phi$ OTDR have been optimize so to achieve the required sensitivity and resolution. Temperature measurement with accuracy of $0.01^{\circ} \mathrm{C}$ and spatial resolution of $1 \mathrm{~m}$ over $8 \mathrm{~km}$ have been reported.

The main application of $\phi$-OTDR is, however, vibration sensing, in particular for intrusion detection [63-73, 82, 181]. Indeed, if the constraint on spatial resolution is relaxed (and it can be relaxed when the target is a several kilometer long fiber), then long optical pulses can be used to probe the fiber, so that the SNR of a single measure can be increased to a level where aver aging is not required. As a result, $\phi$-OTDR can distributedly monitor vibrations along a fiber over a frequency band that is limited only by the roundtrip time, i.e. only by the fiber length. In fact, $1 \mathrm{~km}$ of fiber corresponds to a roundtrip time of about $10 \mu \mathrm{s}$, which yields, by Nyquist theorem, a maximum bandwidth of $50 \mathrm{kHz}$. This means that this technology can be used to implement distributed acoustic sensors with spatial resolution in the order of meters and 
measurement range up to several kilometers-an idea that has already found more than one commercial applications.

\section{DOFS BASED ON POLARIZATION VARIATION}

In principle, an ideal monomodal fiber preserve the polarization of light. This is a consequence of the perfect cylindrical symmetry of the ideal fiber, and is mathematically represented by the two-fold degeneracy of the fundamental mode of propagation [117]. Of course, real fibers do not have a perfect symmetry and hence polarization varies as light propagates in the fiber. The symmetry of the fiber can be broken both because of production imperfections (so called intrinsic causes) and as a consequence of external perturbations (extrinsic causes), that can introduce an asymmetry either geometrical of electromagnetic. These perturbation may be, for example, bending or twist, pressure or strain, magnetic or electric field [182-184]. As a consequence, polarization of light in optical fiber is potentially sensitive to many different external physical fields, and can be used to convert a fiber into a sensor. This approach had been already widely explored in transmission OFS when A.J. Rogers in 1980 proposed the idea of a polarization OTDR (P-OTDR), basically a polarization sensitive OTDR to distributedly measure polarization variation along the fiber for sensing purpose $[86,185]$.

Despite OTDR was proposed in 1976 by Barnosky and Jensen $[98,186]$, its first proposal as a distributed sensor was made by Hartog et al. only in 1983 [128]. Raman-based DOFSs have been publicly proposed not earlier than 1984 by Rogers himself [187] and in 1985 by Dakin et al. [188] (Dakin, however, filed a patent in 1983 [189]), whereas Brillouin-based DOFSs were first proposed in 1989 [190, 191]. As a consequence, P-OTDR is actually the first DOFS ever proposed. Paradoxically, it is the only one which has not yet found a convincing commercial application. While Rogers' original idea was sound and ushered in research activity on DOFSs, it soon turned out that exploiting the full potential of light polarization was not easy at all.

Two main problems hindered the development of polarization-based DOFSs. First, intrinsic fiber asymmetries induce polarization variation on a rather short scale (potentially down to centimeters even for standard telecommunication fibers); therefore, spatial resolution in P-OTDR is constrained by fiber properties and spatial arrangement, rather than by the targeted resolution on the physical field under monitoring. This arises some technical difficulties. Second, the measured quantity, i.e. the polarization of the backscattered light, is not directly related to the local properties of the fiber, but it is affected by the whole roundtrip propagation. Consequently, an inverse scattering problem has to be solved, and this requires an accurate theoretical model and proper data analysis algorithms.

As a consequence, after few examples of P-OTDR as a tool to implement a DOFS were made [192-194], most of the attention turned to the use of P-OTDR (or more in general of polarization sensitive reflectometry) to the characterization of polarization properties of telecommunication fibers. Actually, in the ' 90 s and early years of 2000 , it was clear that the so called polarization mode dispersion (PMD), a phenomenon related to fiber asymmetries, was one of the main obsta- cles to the increase of transmission capacity [195]. In this frame work, P-OTDR was used to analyze the quality of a fiber link, eventually detecting the worst sections [196-205]. Since PMD is a random phenomenon, fibers were characterized in terms of statistical properties, and P-OTDR was used to measure average quantities. This allowed to substantially relax constraints on spatial resolution, and enabled the use of simplified theoretical models and data analysis algorithms.

After some years of inactivity, however, the increasing interest in DOFSs, the improvement of opto-electronic technology, and the development of novel data analysis algorithms, revived attention on polarization-based DOFSs [177180, 206-224]. While a commercial solution is still not available to date, polarization-based DOFSs have unique and promising features (such as sensitivity to magnetic field) that deserve consideration.

\subsection{Practical Implementations}

The aim of a polarization sensitive reflectometer (PSR) is to measure the SOP variation of the backscattered light, as a function of scattering position. The measurement may retrieve partial or complete information; correspondingly, complexity of experimental setup and of measurement procedure increases. Fundamentally, there are two different approaches, shown in Fig. (9).

In the first case [see Fig. (9a)] a polarizer is interposed between the reflectometer and the sensing fiber, so to polarize both the probe light and the backscattered one. In this way, variations of the backscattered SOP are converted in power fluctuations, which are measured by the reflectometer. As described below more in detail, the spatial frequency of these fluctuations is related to local birefringence strength. This approach, simple as it is, is not able however to retrieve the complete information carried by the backscattered SOP.

The second approach consists in measuring the complete SOP of backscattered light, possibly for more than one different input SOP. To perform this kind of measurement, it is necessary to insert a polarization controller in the input path and a polarization analyzer in the output one. A possible implementation based on a standard reflectometer is shown in Fig. (9b); if the reflectometer is custom built, the polarization controller and the polarization analyzer can be inserted after the light source and before the photo-receiver, respectively. The polarization analyzer can be implemented as a cascade of a rotating quarter wave-plate followed by a fixed polarizer [225]. Measurements are then repeated for at four different orientations of the quarter wave-plate; this provides enough information to calculate the SOP of the backscattered light. When the reflectometer is an OFDR with a polarization sensitive receiver, the polarization analyzer can be omitted, because (in principle) the backscattered SOP can be measured by the OFDR receiver. Measuring the backscattered SOP for more than one input SOP allows a complete characterization of the polarization transformation induced by roundtrip propagation (i.e. its Jones or Mueller matrices). Nevertheless, it is worthwhile remarking that in order to take the most out of this complete characterization it is necessary to resort to a rather complex data analysis procedure, described below. Actually, the raw information provided by the backscattered SOP is related to the cumulative effect induced 


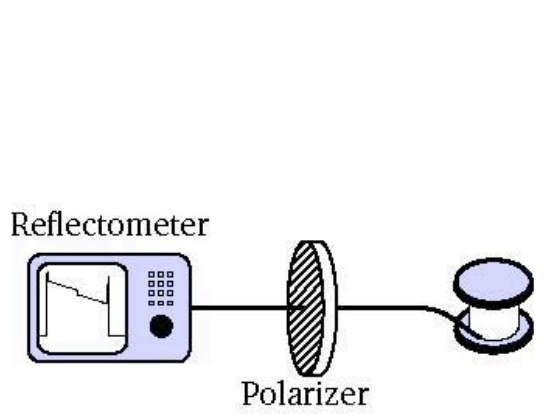

(a)

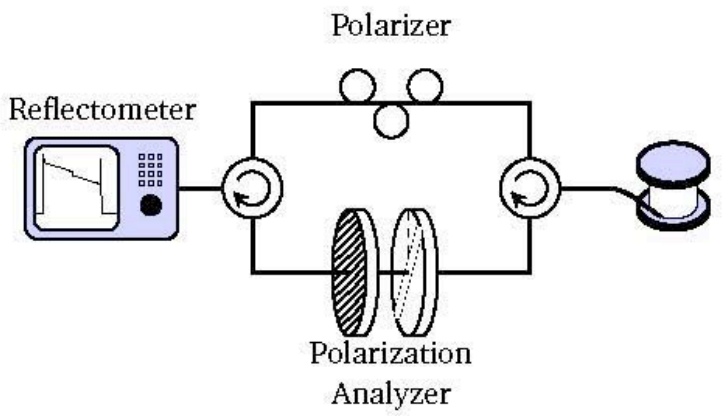

(b)

Fig. (9). Possible approaches to polarization-based DOFSs. (a) A polarizer is interposed between the reflectometer and the fiber to convert SOP variations of the backscattered light in power fluctuations. (b) A polarization controller and a polarization analyzer are inserted in the input and output paths, respectively; this allows a complete polarimetric characterization of roundtrip propagation.

by roundtrip propagation in the fiber, while its relationship with fiber local properties is not trivial.

In both approaches the reflectometer can be of any type; OTDR, $v$-OTDR and OFDR have been used, so far. In the case of time domain techniques, however, the probe pulse must be narrow-band, otherwise PMD may depolarize the pulse and, consequently, the backscattered light. The performance of PSR depends largely on the employed reflectometer. OTDR-based PSR may interrogate kilometerslong fibers, with spatial resolution hardly below half a meter [201], where as $v$-OTDR-based PSR may achieve centimeter-scale resolution, but on much shorter distance [223]. Subcentimeter spatial resolution can be achieved with OFDRbased PSR, but only on distance range of few tens of meters [226-228]. P-OFDR measurements with spatial resolution in the order of tens of centimeters over kilometers long fibers have been reported, although not for sensing applications [204].

One of the main issues of PSR is the rather long measurement time, especially for the second approach [Fig. 9b)] where the raw acquisition has to be repeated for different orientations of the polarization analyzer and for different in put SOPs. The complete measurement of the backscattered SOP may take as long as few minutes, therefore polarizationbased DOFSs are most befitted for the sensing of static physical fields.

\subsection{Theoretical Model}

As anticipated above, the correct interpretation of data provided by polarization based DOFSs requires an accurate theoretical modeling of roundtrip propagation.

Limiting the analysis to fiber not affected by polarization dependent loss (which is by far the most common case), the polarization of light propagating in a fiber can be conveniently represented in the 3-dimensional space of unit Stokes vectors [225]. ${ }^{1}$ Let $\hat{s}_{0}$ be the unit Stokes vector representing input polarization; the polarization at a given position $z$ along the fiber then reads $\hat{s}(z)=\boldsymbol{F}(z) \hat{s}_{0}$, where $\boldsymbol{F}(z)$ is the $3 \times 3$ Mueller matrix representing forward propagation up to $z$. Owing to the supposed absence of polarization dependent loss, Mueller matrices are orthogonal and represent rotations

${ }^{1}$ According to the notation customarily accepted in optics, in this paper-right hand circular polarization is represented by the third axis of the Stokes space. in the Stokes space. Consequently, they have unitary determinant and their inverse is equal to their transpose, i.e. $\mathbf{F}^{-1}=$ $\mathbf{F}^{\mathrm{T}}$.

Neglecting issues related to phase interference, we assume that Rayleigh scattering takes place continuously along the fiber, so that backscattered light is originated at each $z$. In general, the state of polarization (SOP) of the light that has under gone a roundtrip (i.e. forward propagation up to $z$, scattering and backpropagation to the fiber in put) can be expressed as $\hat{s}_{R}(z)=\boldsymbol{B}(z) \boldsymbol{S} \boldsymbol{F}(z) \hat{s}_{0}$, where $\boldsymbol{B}(z)$ is the Mueller matrix representing backward propagation from $z$ to the fiber input, and $\boldsymbol{S}$ is the Mueller matrix representing Rayleigh backscattering.

An important aspect of this analysis is the reference frame. When describing polarization of light, it is custom to express the SOP with respect to a reference frame in which the third coordinate corresponds to the direction of propagation [117]. In the present case, however, since we consider two opposite directions of propagation, it is much more convenient to express all the analysis with respect to a single reference frame. Therefore, hereinafter, all the equations will be referred to a right-hand reference frame, whose third axis, $\mathrm{z}$ corresponds to the direction of forward propagation. With respect to this frame, the Mueller matrix of Rayleigh backscattering, neglecting losses, is simply the identity; therefore

$\hat{s}_{R}(z)=\mathbf{B}(z) \mathbf{F}(z) \hat{s}_{0}=\mathbf{R}(z) \hat{s}_{0}$,

where $\boldsymbol{R}(z)$ is the Mueller matrix of roundtrip propagation. It may be shown that if the fiber is reciprocal, i.e. if it is not exposed to magnetic field oriented with the fiber axis, then $\boldsymbol{B}=\boldsymbol{M} \boldsymbol{F} \boldsymbol{M}$, where $\boldsymbol{M}=\operatorname{diag}(1,1,-1)$ is a diagonal matrix [196]. This property is a direct consequence of Lorentz reciprocity theorem $[117,229]$; ho wever, as we will see, it is not strictly needed.

The variation of light polarization along the fiber can be described also by means of the differential equation

$\frac{d \hat{s}}{d z}=\bar{\beta}(z) \times \hat{s}(z)$,

here the real vector $\bar{\beta}(z)=\left(\beta_{1}, \quad \beta_{2}, \quad \beta_{3}\right)^{T}$ is the birefringence vector, and is related to $\boldsymbol{F}(z)$ by the expression $\bar{\beta} \times=(d \boldsymbol{F} / d z) \boldsymbol{F}^{\mathrm{T}}$; this yields also 


$$
\frac{d \mathbf{F}}{d z}=\bar{\beta}(z) \times \mathbf{F}(z)
$$

The birefringence vector summarizes all the effects that perturbations acting in a given point of the fiber have on its polarization properties. It can be decomposed in linear birefringence, $\bar{\beta}_{L}=\left(\beta_{1}, \quad \beta_{2}, 0\right)^{T}$ and circular birefringence $\bar{\beta}_{C}=\left(\begin{array}{lll}0, & 0, \beta_{3}\end{array}\right)^{T}$. Linear birefringence is caused, for example, by anisotropy, bending or geometrical asymmetries. Differently, circular birefringence can be induced only by twist or magnetic field [182]. ${ }^{2}$

Whenever a fiber is twisted, two effects take place: (i) the intrinsic linear birefringence is rotated (simply because the fiber itself is rotated), (ii) a reciprocal circular birefringence (optical activity) proportional to the twist rate is induced $[182,212]$. Specifically, the birefringence vector of a twisted fiber can be written as:

$$
\bar{\beta}(z)=\mathbf{U}[2 \tau(z)] \bar{b}(z)+\left(\begin{array}{c}
0 \\
0 \\
-g(d \tau / d z)
\end{array}\right)
$$

where $\bar{b}(z)=\left(b_{1}, \quad b_{2}, \quad 0\right)^{T}$ is the intrinsic birefringence vector of the untwisted fiber, $\tau(z)$ is the angle by which the fiber is rotated at $z$, and

$$
\mathbf{U}(\theta)=\left(\begin{array}{ccc}
\cos \theta & -\sin \theta & 0 \\
\sin \theta & \cos \theta & 0 \\
0 & 0 & 1
\end{array}\right)
$$

represents rotation by an angle $\theta$ around the third axis of Stokes space, $\hat{\mathrm{s}}_{3}$, which corresponds to physical rotation by an angle $\theta / 2$ around the axis $\hat{z}$.

Differently, when the fiber is exposed to a static magnetic field nonreciprocal circular birefringence is induced. Actually, nonnegligible magnetic field parallel to the direction of propagation causes Faraday rotation of polarization $[182,230]$; hence, the birefringence vector reads [220]

$$
\bar{\beta}(z)=\bar{b}(z)+\left(\begin{array}{c}
0 \\
0 \\
2 V B(z) \cos \psi(z)
\end{array}\right),
$$

where again $\bar{b}(z)$ is the intrinsic birefringence, $B(z)$ is the amplitude of magnetic induction, $\psi(z)$ is the angle subtended by magnetic induction and direction of forward propagation, and $V$ is the Verdet constant. For Ge-doped silica fibers $V$ is about $0.6 \mathrm{rad} \mathrm{T}^{-1} \mathrm{~m}^{-1}$ at $1550 \mathrm{~nm}[231,232]$; therefore, rather intense magnetic fields or large fiber lengths are needed to observe nonnegligible polarization rotation.

The difference between reciprocal and nonreciprocal birefringence manifests itself when considering backward propagation. In fact, it can be shown that, similarly to Eq. (4), the differential equation describing the Mueller matrix of backward propagation reads [220]:

${ }^{2}$ Given the expression $\bar{a} \times \bar{b}$, simple algebraic considerations allow to consider the quantity (ax) as a linear operator, whose expression is a $3 \times 3$ skew-symmetric matrix. $\frac{d \mathbf{B}}{d z}=\bar{\gamma}(z) \times \mathbf{B}(z)$,

where, in the most general case,

$\bar{\gamma}(z)=-\mathbf{U}[2 \tau(z)] \bar{b}(z)+\left(\begin{array}{c}0 \\ 0 \\ -g(d \tau / d z)\end{array}\right)-\left(\begin{array}{c}0 \\ 0 \\ 2 V B(z) \cos \psi(z)\end{array}\right)$.

Note that, with respect to Eqs. (5) and (7), in this expression all terms have changed sign, except reciprocal twistinduced circular birefringence.

Taking the $z$-derivative of (2), and exploiting Eqs. (5), (7) and (9), it can be showed that

$$
\begin{aligned}
& \frac{d \hat{s}_{R}}{d z}=\bar{\beta}_{R}(z) \times \hat{s}_{R}(z), \\
& \frac{d \mathbf{R}}{d z}=\bar{\beta}_{R}(z) \times \mathbf{R}(z),
\end{aligned}
$$

where the roundtrip birefringence vector reads

$$
\bar{\beta}_{R}(z)=2 \mathbf{B}(z)\left[\bar{b}_{L}(z)+\left(\begin{array}{c}
0 \\
0 \\
2 V B(z) \cos \psi(z)
\end{array}\right)\right],
$$

$\bar{\beta}_{L}$ being the linear component of forward birefringence. Clearly roundtrip birefringence does not have any explicit dependence on reciprocal circular birefringence. Note however, that the effects of this birefringence are still present in $\boldsymbol{B}(z)$. According to Eq. (12), the roundtrip birefringence is equal to twice the forward birefringence (excluded the reciprocal circular term) "seen through" the backpropagation matrix.

Equations (10)-(12) show that the variation of the roundtrip birefringence, which is the quantity measured by polarization-based DOFS, is profoundly different from that of forward birefringence, which is the one we would actually want to measure. To give an idea of how different this variations can be, Fig. (10a) shows the simulated variation of $\hat{s}(z)$ along a reciprocal randomly birefringent fiber, whereas Fig. (10b) shows the simulated variation of polarization of the corresponding backscattered light. It can be proved that the accumulation point is related to the input SOP. Proper analyses are therefore, necessary to extract from $\hat{s}_{R}(z)$ the sought local information.

\subsubsection{Extracting Local Information}

The problem of extracting local information from the measured backscattered SOP, $\hat{s}_{R}(z)$ is due to the presence of matrix $\boldsymbol{B}(z)$ in the expression $\bar{\beta}_{R}(z)$. Actually, that matrix is unknown, therefore linear birefringence and Faraday rotation cannot be determined straightforwardly. Nonetheless, $\boldsymbol{B}(z)$ is by definition orthogonal, thus it preserves the modulus of vectors.

As a consequence

$$
\beta_{R}(z)=\left|\bar{\beta}_{R}(z)\right|=2 \sqrt{\beta_{L}^{2}(z)+[2 V B(z) \cos \psi(z)]^{2}},
$$




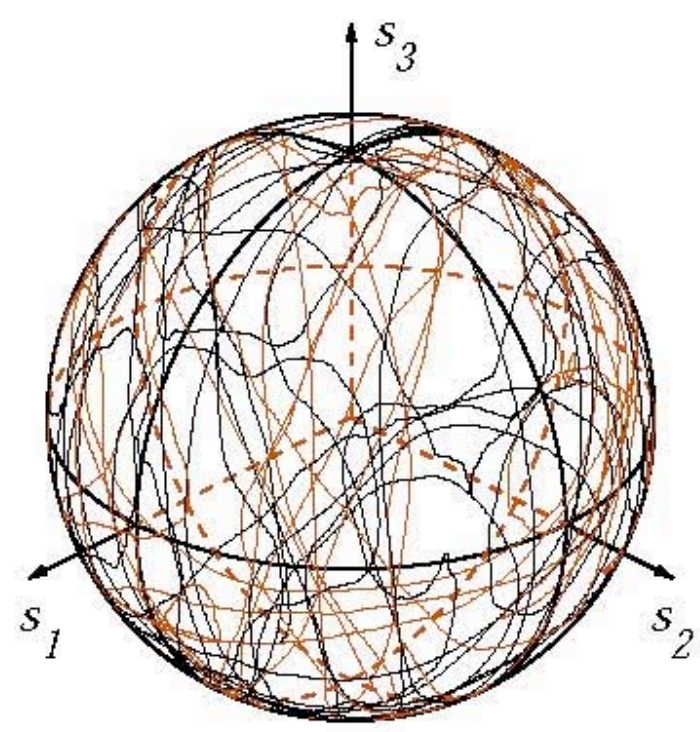

(a)

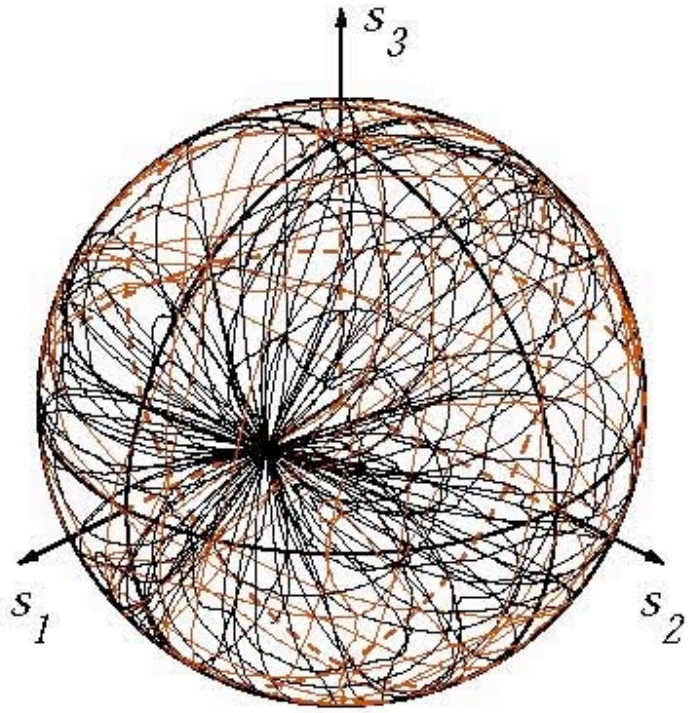

(b)

Fig. (10). Simulated trajectories of SOP of light propagating in a randomly birefringent fiber. (a) SOP of forward propagating light. (b) SOP of the corresponding roundtrip light.

and noting that the modulus of $d \hat{s}_{R} / d z$ is proportional to the modulus of $\bar{\beta}_{R}(z)$, we may conclude that the frequency of local variation of the backscattered SOP is proportional to the local properties of the fiber. Particularly interesting are the cases in which either linear birefringence or (more commonly) Faraday rotation is negligible, for in this case the frequency of variation of $\hat{s}_{R}(z)$ is proportional to only one of the effects. This is the working principle of the first practical approach described above and shown in Fig. (9a). Generally, the analysis consists in a sliding FFT on the recorded power. The simplicity of the approach comes at the cost of reduced accuracy and partial lost of information. Actually, no information about the direction of birefringence can be retrieved. Furthermore, the frequency of variation of $\hat{s}_{R}(z)$ depends not only on $\beta_{R}(z)$, but also on the angle subtended by $\hat{s}_{R}(z)$ and $\beta_{R}(z)$. Depending on fiber type, this angle may vary quite rapidly and in such case the simple relationship between $\beta_{R}(z)$ and the local frequency content of $\hat{s}_{R}(z)$ may fade away.

All the information carried by $\hat{s}_{R}(z)$ can be extracted by solving the complete inverse scattering problem. The demonstration of the procedure would be out of scope, therefore, the interested reader is deferred to the literature [220]. Here we just report the final result. In fact, the procedure is made of the following steps:

(1) the roundtrip SOP, $\hat{s}_{R}(z)$, is measured for at least two different input SOPs;

(2) from the information gathered at step 1, the roundtrip birefringence vector, $\bar{\beta}_{R}(z)$, can be calculate exploiting procedures known in the literature [233, 234];

(3) the following differential equation is numerically solve:

$\frac{d \mathbf{Q}}{d z}=\frac{1}{2} \bar{\beta}_{R}(z) \times \mathbf{Q}(z)$, with $\quad \mathbf{Q}(0)=\mathbf{I} ;$ it may be shown that in a proper reference frame, rotating along the fiber at a rate equal and opposite to the applied twist, $\boldsymbol{Q}(z)$ is equal to the transpose of the backward matrix $\boldsymbol{B}(z)$;

(4) the apparent birefringence vector is finally calculated as:

$\bar{\beta}_{A}(z)=\frac{1}{2} \mathbf{Q}^{T}(z) \bar{\beta}_{R}(z)$.

the key point of this procedure is that it can be shown that the apparent birefringence vector calculated as above reads [220]

$\bar{\beta}_{A}(z)=\mathbf{U}[(2-g) \tau(z)] \bar{b}(z)+\left(\begin{array}{c}0 \\ 0 \\ 2 V B(z) \cos \psi(z)\end{array}\right)$.

This expression has two important aspects that deserve attention. First, since $\bar{b}(z)$ is linear by definition, the third component of $\bar{\beta}_{A}(z)$ depends exclusively on Faraday rotation. Spoken in other words, the above procedure allows to measure the magnetic field parallel to the fiber distributedly as a function of $z$.

Second, the linear component of $\bar{\beta}_{A}(z)$ allows to determine both modulus and direction variation of the fiber intrinsic birefringence. This enables, for example, the distributed measurement of the angle by which the fiber is rotated as a function of $z$, which yields a rather unique twist sensor.

The analysis reported above has neglected, for simplicity, the effects of the output patch cord that is typically interposed between the fiber input and the polarization analyzer [see Fig. (9b)]. Owing to this patch cord, the reference frame is rotated in an unknown way, shuffling components of 

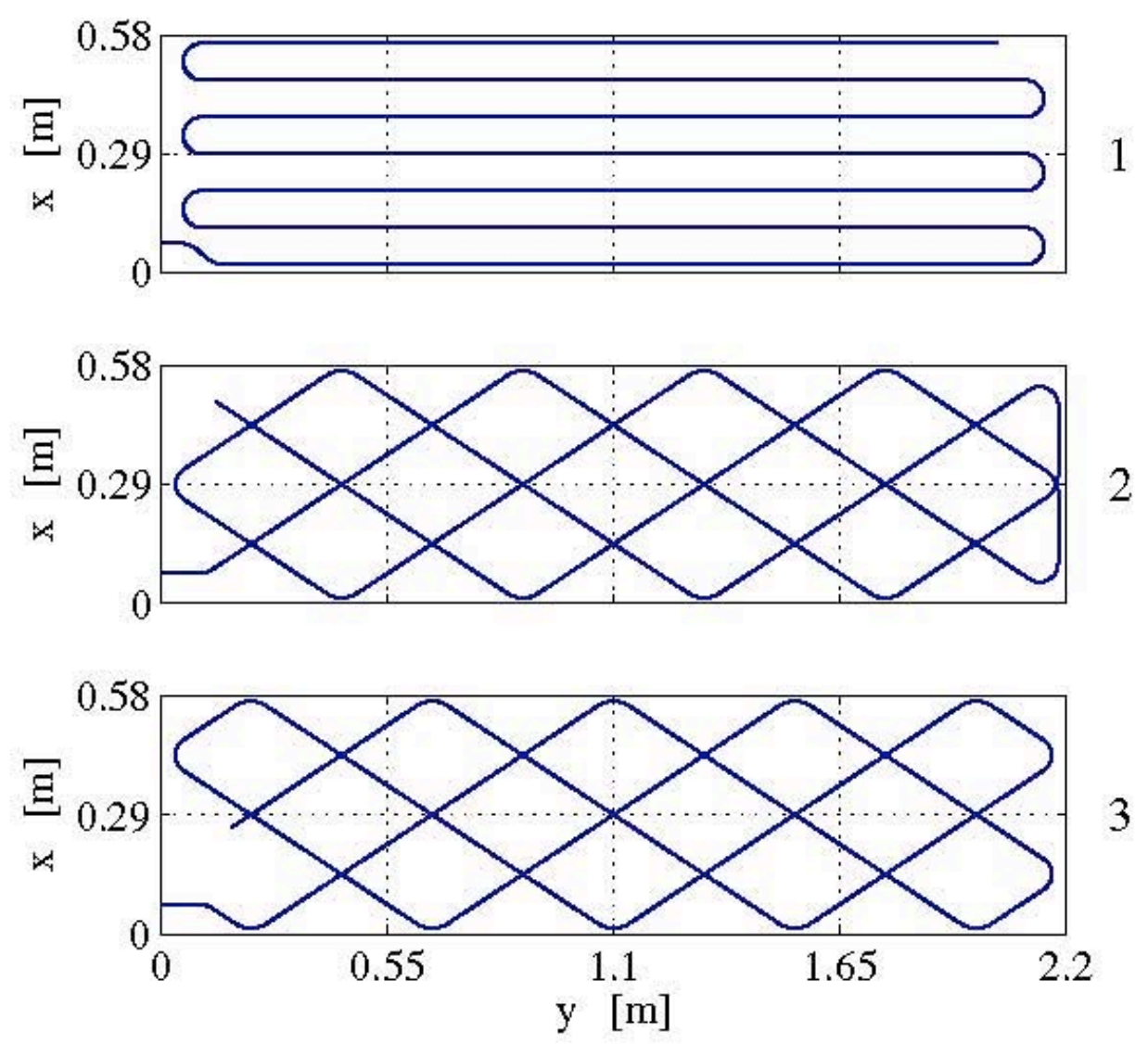

Fig. (11). Path along which the three fibers of the magnetic field sensor are laid.

$\bar{\beta}_{A}(z)$ so that the third one is no longer strictly related to circular birefringence. This unknown rotation can be taken into account by a proper calibration. Alternatively, it can be compensated numerically, exploiting the fact that when there is no Faraday rotation $\bar{\beta}_{A}(z)$ lays on a plane. In this case, however, the absolute direction of rotation and the absolute direction of the tangent magnetic field cannot be determined without a known reference. Nonetheless, relative variations are still correctly tracked $[212,220]$.

\subsection{Examples of Application}

The application of polarization-based DOFSs to different fields, ranging from temperature to magnetic fields, has been explored in several research papers. Below were view the main results.

\subsubsection{Magnetic Field}

The idea of using a P-OTDR to measure magnetic field was first explored experimentally by Ross in 1981 [192]. That work is actually the first attempt to experimentally implement a DOFS; however, the implemented set up was only able to detect the point were the magnetic field was applied along the fiber, assuming there were only one of such points. After that experience, the idea has been abandoned for almost 30 years, and only recently it has been developed in to a real distributed sensor [220-224]. The working principle, based on Faraday rotation, has been described in Sec.5.2.1.
An example of application has been reported in Ref. [224]. The sensor consisted in 3 fibers laid on a wooden support along the paths shown in Fig. (11). Faraday rotation has been measured along each of these fibers using a P-OFDR similar to that described in Fig. (9b); in this way, the magnetic field in the area spanned by the sensor was sampled at different points and along different directions. The sensor has been tested in a magnetic resonance imaging (MRI) scanner for medical applications. The scanner has a magnetic field of $1.5 \mathrm{~T}$, parallel to the bore axis and to the long side of the sensor. Fig. (12) shows for example the apparent birefringence vector, $\bar{\beta}_{A}(z)$, measured along the first fiber. We note that, while the first two components vary almost randomly, reflecting the random nature of linear birefringence, the third component has a deterministic evolution exactly corresponding to the fact that the fiber periodically changes direction with respect to the magnetic field. ${ }^{3}$

Exploiting the fact that the three fibers were laid one over the other, it has been possible to use the measurements gathered along each fiber to calculate a vector map of the magnetic field projection on the sensor plane. The result is shown in Fig. (13). This preliminary test, performed on standard G.652 fibers, achieved a relative accuracy of about 7\%, with a spatial resolution of about $3 \mathrm{~cm}$; the noise floor was around $100 \mathrm{mT}$.

\footnotetext{
${ }^{3}$ Kim et al. explored the same concept in 1982 [194]. However, despite the title, their work is based on the analysis of Frenel reflection from the fiber far end; the possibility of using Rayleigh scattering is just mentioned in a marginal note.
} 

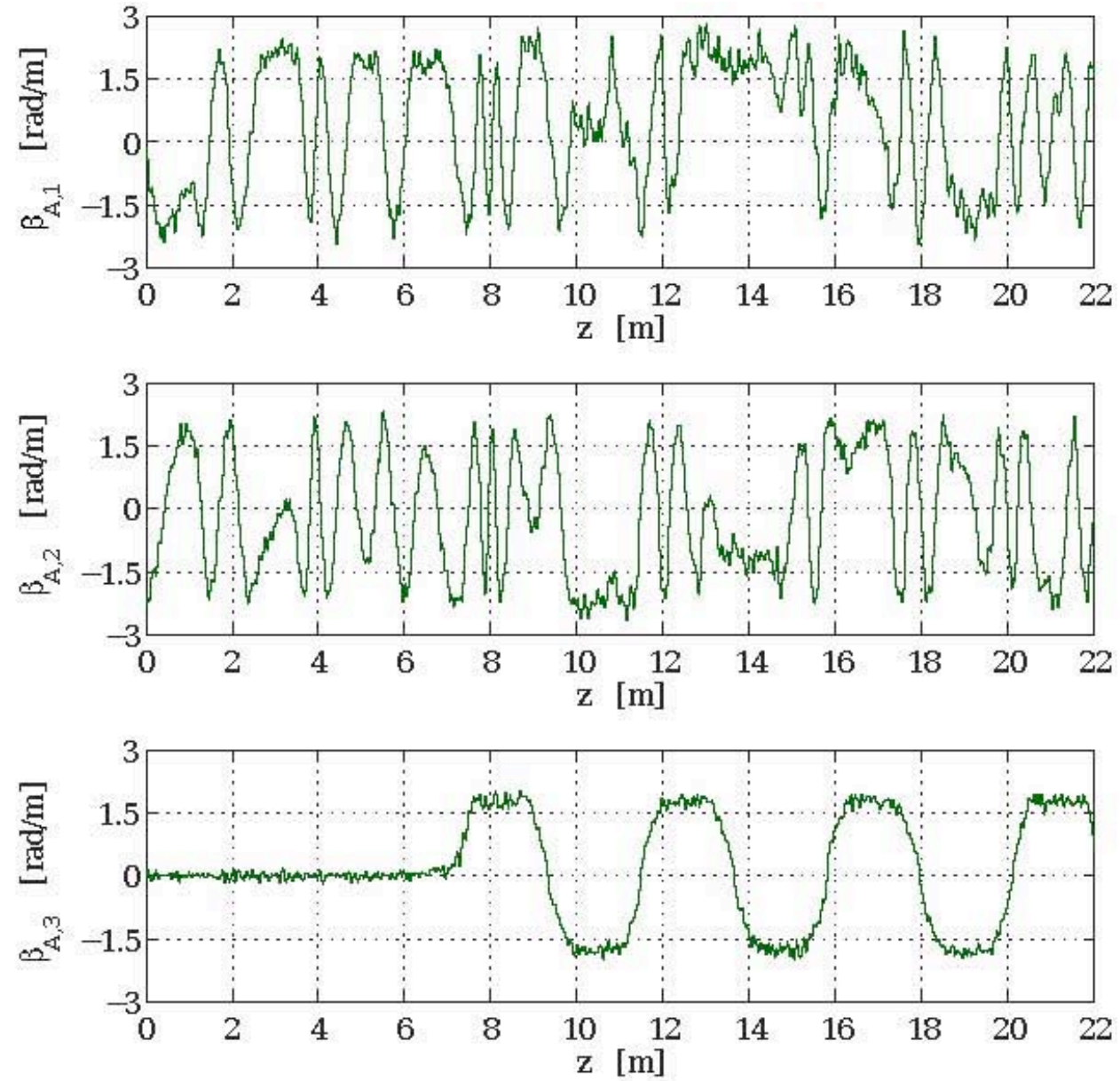

Fig. (12). Components of the apparent birefringence vector measured along fiber of Fig. (11). The first 7-m-long section corresponds to the fiber connecting the reflectometer to the sensor.

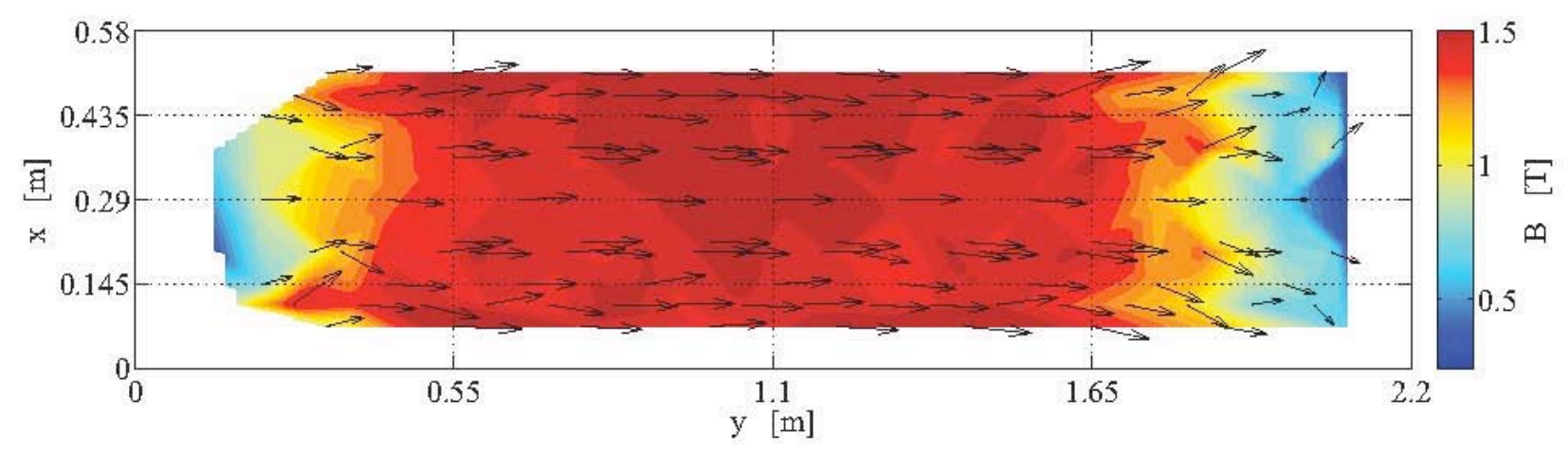

Fig. (13). Vector map of the projection of magnetic field on the sensor plane.

We remark that the ability of performing distributed measurement of magnetic fields is very unique to polarization-based DOFSs, and so far has not been paralleled by other distributed techniques.

\subsubsection{Twist}

Another unique ability of polarization-based DOFSs is the possibility of measuring the twist applied to the fiber, i.e. the angle by which the fiber is rotated at each point [216, 217]. This sensor is based on the fact that when the fiber is twisted, its intrinsic linear birefringence undergoes the same rotation; therefore, the twist angle can be measured by measuring the direction of linear birefringence. As an example, Fig. (14) shows the linear birefringence rotation measured along a fiber that was laid straight on the laboratory floor, fixed at one end and twisted at the other at known rates. Clearly, the technique has been able to correctly measure the effects of twist; the slow oscillations are due to the intrinsic spin applied to the fiber during production. The accuracy of the measured rotation has been estimated to be about $3^{\circ}$. 


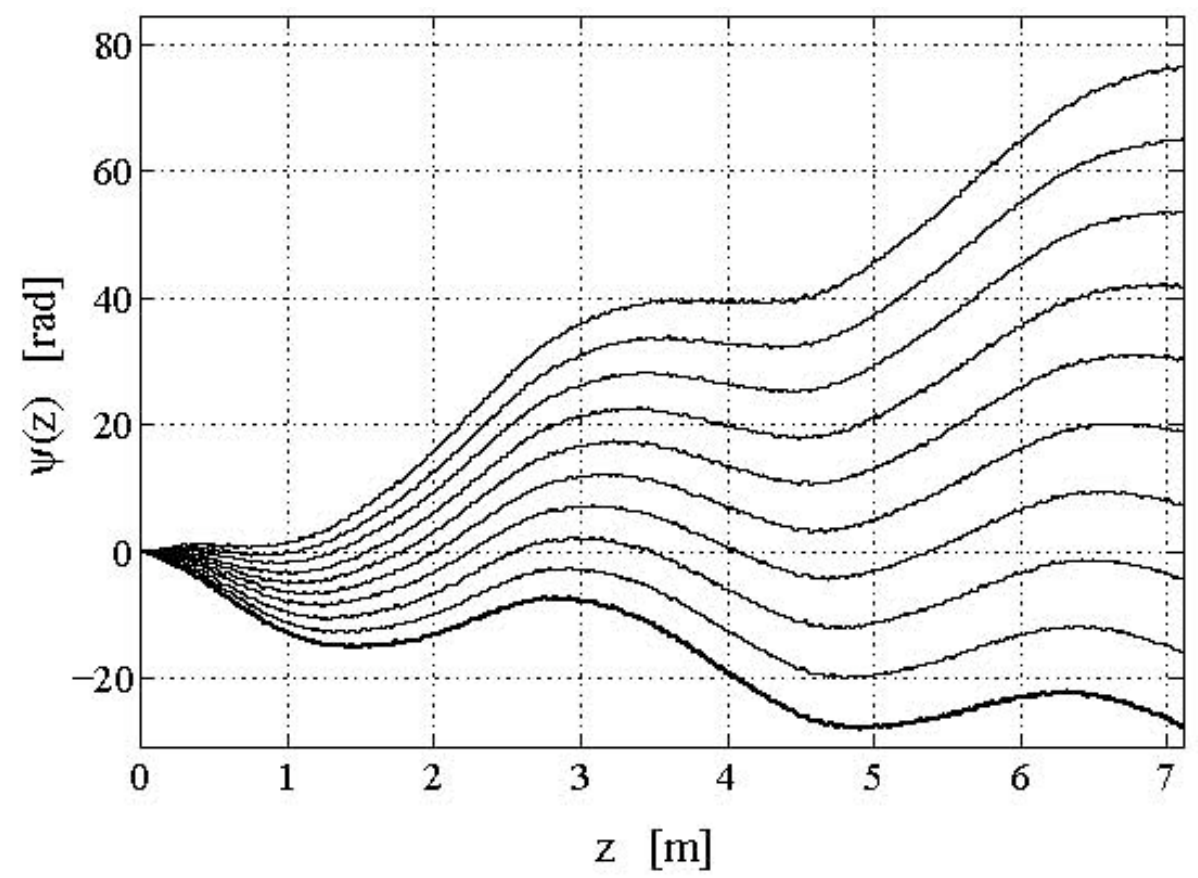

Fig. (14). Angle of rotation measured along a fiber uniformly twisted at different rates.

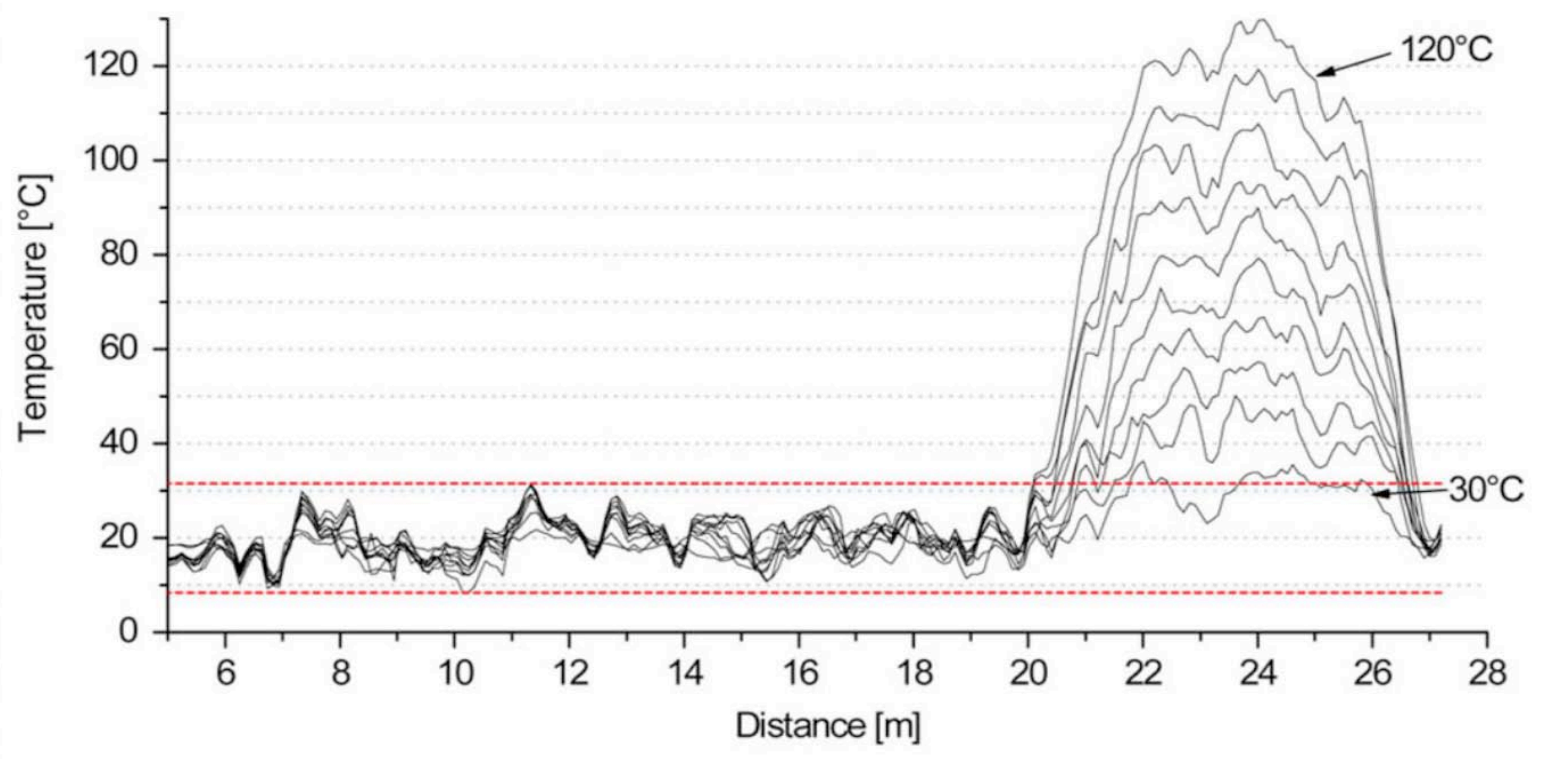

Fig. (15). Example of temperature measurement. Reprinted with permission from Ref. [180].

\subsubsection{Temperature}

The most convincing example of temperature measurement by means of a polarization-based DOFS has been reported by Crunelle et al. [180]. The sensor exploits the setup shown in Fig. (9a), and is based on the fact that fiber birefringence is linearly dependent on temperature. An example of result is shown in Fig. (15). The sensor is able to detect temperature variation of $10^{\circ} \mathrm{C}$ with a spatial resolution of $1.5 \mathrm{~m}$; furthermore, hot spot of $120^{\circ} \mathrm{C}$ has been detected with a resolution of $3 \mathrm{~cm}$. While Brillouin- and Raman-based systems achieve better resolution, the proposed polarizationbased DOFS is indeed simpler.
A different approach has been proposed by Donlagic and Lesic [179]. The setup is again similar to that of Fig. (9a), but now the sensing elements are short sections of polarization maintaining fiber inserted along the link. The sensor, which is actually quasi-distributed, can interrogate 20 of such sensors with a reported temperature accuracy of $0.1^{\circ} \mathrm{C}$.

\subsubsection{Others}

Other fields of application have been proposed and marginally explored. The use of polarization-based DOFSs to measure strain has been discussed by some authors [177, 


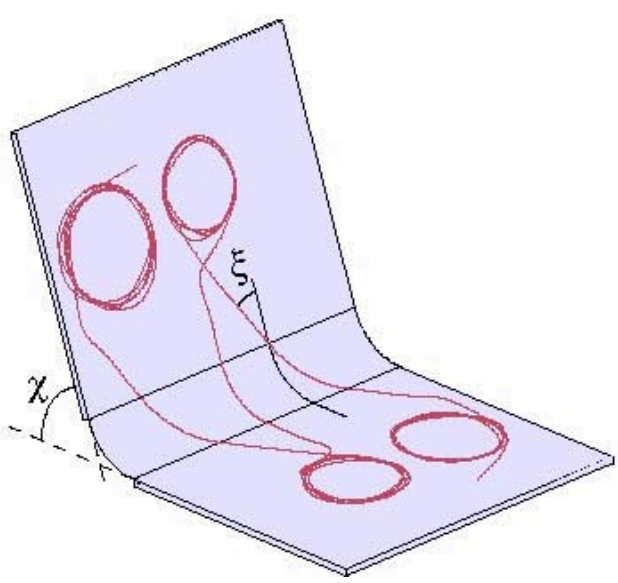

(a)

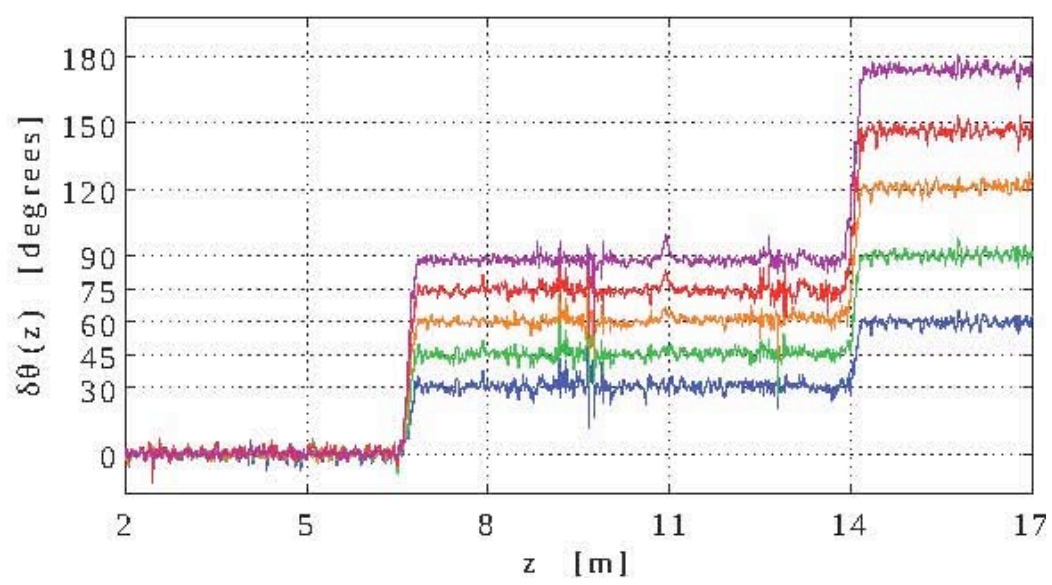

(b)

Fig. (16). Example of geometrical sensing. (a) Foldable structure over which the fiber is deployed. (b) Angle of rotation measured along the fiber for different fold angles, $\chi$. Vertical values are the nominally expected ones.

178, 206-209]. However, results seem not to be up to the performance of Brillouin or FBG-based systems.

Similarly, a few works have studied the use of polarization-based DOFSs for vibration monitoring. Zhang and Bao proposed a sensor based on a P-OTDR. The device, however, is notable to distinguish two sources of vibration when they act simultaneously, because the first one in space would hide the following ones. This problem is solved, in principle, in Ref. [211, 212], where it has been shown that, by using the complete setup of Fig. (9b), it is possible to isolate information from each vibrating fiber section. The method is however based on the complete polarimetric characterization of the fiber, which is quite time demanding; therefore, the method can be applied only to very low frequency vibrations.

Rogers et al. explored also the possibility of using a polarization-based DOFS to measure pressure [210]. While in principle a standard fiber could have been used, in order to increase sensitivity, a special fiber with an air hole parallel to the core was used in this application. This hole gives flexibility to the fiber, which becomes much more sensitive to pressure and lateral loads. The sensing was achieved by measuring fiber birefringence.

Finally, it is worthwhile mentioning the use of a P-OFDR to sense geometrical properties of the fiber path [218]. The idea is that when the fiber is laid on a non co-planar path it necessarily undergoes a twist. Measuring this twist allows to infer information on the geometry of the path. This simple explanation hides however some very subtle aspects. In fact, the problem is determining the reference frame with respect to which the birefringence orientation is measured.

Considerations about parallel transport of light polarization [235-237], which is intimately related to Berry's phase [238], allows to conclude that the reference frame is that defined by the polarization analyzer parallel transported along the fiber [218]. The idea has been tested with the structure shown in Fig. (16a), made of two flat panels connected by a flexible surface, so that it can be folded much like a book. A fiber is laid on the structure in such a way that it crosses the flexible surface at an angle with respect to the fold axis. As a result, when the structure is folded, those fiber portions undergo a rotation related to the fold angle. Results of the measurements performed for different angles are shown in Fig. (16b) and are in good agreement with the expected nominal values.

\section{CONCLUSIONS}

The idea that Rogers put forth in 1980, of using polarization of Rayleigh backscattered light to implement a fiber optic sensor able to distributedly measure various physical fields along the fiber, opened the way to the development of DOFSs. Since then, more than 200 papers have been published on international journals and conferences proceedings about Rayleigh-based DOFSs, and the rate of publications per year has a tendency to grow [see Fig. 1]. For completeness, we have tried to include an exhaustive list in the bibliography, and to facilitate its use we have classified contributions with respect to their field of application. Results are shown in Table $\mathbf{1}$.

During the three decades elapsed since the first proposal, Rayleigh scattering has been exploited in different solutions to sense quantities as diverse as temperature and chemicals concentration, vibration and magnetic field. Among the explored approaches, the most successful has been that based on the analysis of interference fringes created by the discrete nature of Rayleigh scattering centers. These fringes can be measured either in time- or in frequency-domain, yielding to two different classes of DOFSs.

In the first case, measurement speed and long range enable to attain effective vibration sensors, that have application mainly in intrusion and leak age detection. Frequencydomain approach, in contrast, has a shorter range (below hundreds of meters), but achieves extremely high resolution and sensitivity, enabling temperature and strain measurement with a very high number of equivalent sensing points. Both the two approaches have found commercial applications. 
Table1. Classification of Bibliographical References with Respect to their Field of Application and Sensing Mechanism

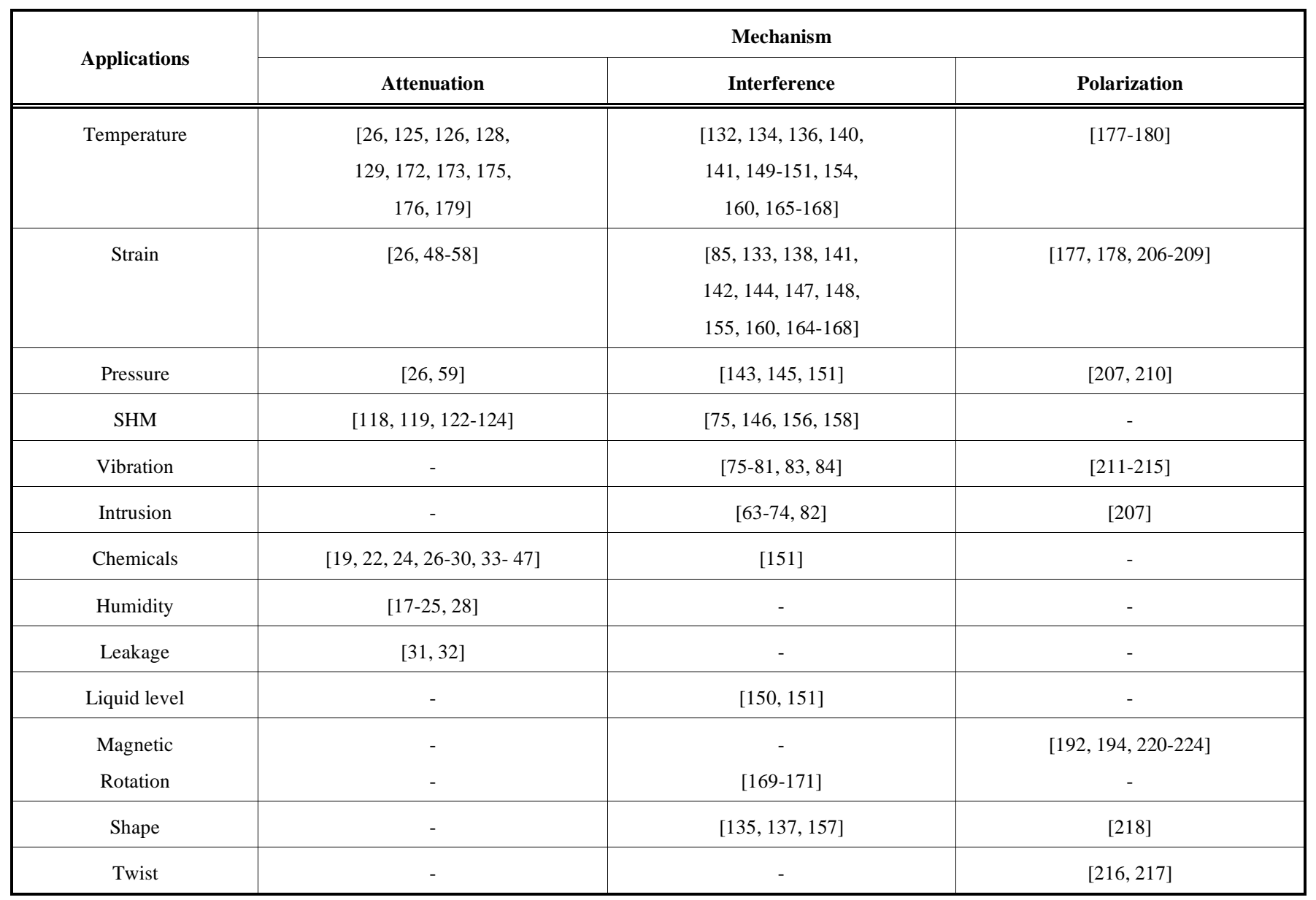

Systems based on attenuation have been studied for rather long time. Their main advantage is, in most cases, simplicity, as they just measure the intensity of the backscattered light. Optical losses are induced by the external physical field by means of proper transducing mechanism, mainly based on micro-bending or interaction through evanescent light. This approach has been successfully exploited to implement DOFSs for various parameters, including different chemicals, humidity and temperature. Owing to their relative simplicity and consequent cheapness, these kind of sensors have found some commercial interest.

The original idea of exploiting polarization of backscattered light turned out to be a rather complex task, to the extent that, a few preliminary tests apart, it took almost 20 years before the technique found application as distributed sensor. Recent developments in opto-electronic equipments and in theoretical modeling of the problem allowed to revive the interest around polarization-based DOFSs. Actually, this class of sensors offers unique properties, unparalleled even by DOFSs not based on Rayleigh scattering. Probably, the most promising of these properties is the recently demonstrated ability of mapping magnetic fields in the area spanned by the fiber.

The first attempt ever made to experimentally implement a DOFS was made by Ross in 1981, and, paradoxically, it was just a magnetic field sensor based on polarization of
Rayleigh-backscattered light. The proposal was quite crude indeed, yet it confirmed that distributed sensors could be made out of optical fibers and that polarization of light could play an important role.

\section{CONFLICT OF INTEREST}

The authors confirm that this article content has no conflicts of interest.

\section{ACKNOWLEDGMENTS}

Authors are indebted to Andrea Galtarossa for fruitful discussions and invaluable support. Partial financial support from Fondazione Cassa di Risparmio di Padova e Rovigo, and from the Italian Ministry of Foreign Affairs (Direzione Generale per la Promozione del Sistema Paese) is acknowledged. This work has been performed under the agreement with ISCTI, Rome, Italy.

\section{REFERENCES}

[1] Culshaw B, Kersey A. Fiber-Optic sensing: A historical perspective. J Lightw Technol 2008; 26(9): 1064-78.

[2] Kingsley SA. Fiber-Optic sensors: Opportunities for distributed measurement. InTech 1985; 32(8): 44- 8.

[3] Kersey AD, Dandridge A. Distributed and multiplexed fibre-optic sensor systems. IEREJ 1988; 58(5): S99-111. 
[4] Rogers A. Distributed optical-fibre sensors for the measurement of pressure, strain and temperature. Phys Rep 1988; 169(2): 99-143.

[5] Rogers A. Distributed optical-fibre sensors for the measurement of pressure, strain and temperature. IEREJ 1988; 58(5): S113-22.

[6] Dakin JP. Distributed optical fiber sensors. In: Proceedings of SPIE - The International Society for Optical Engineering. vol. 1797. Boston: USA 1993. pp. 76-108.

[7] Rogers A. Distributed optical-fibre sensing. Meas Sci Technol 1999; 10(8): R75-99.

[8] Henault JM, Moreau G, Blairon S, et al. Truly distributed optical fiber sensors for structural health monitoring: from the telecommunication optical fiber drawling tower to water leakage detection in dikes and concrete structure strain monitoring. Adv Civil Eng 2010; 2010: 1-13.

[9] Lopez-Higuera JM, Rodriguez CL, Quintela IA, Cobo A. Fiber optic sensors in structural health monitoring. J Lightw Technol 2011; 29(4): 587-608.

[10] Bao X, Chen L. Recent progress in distributed fiber optic sensors. Sensors 2012; 12(7): 8601-39.

[11] Ukil A, Braendle H, Krippner P. Distributed temperature sensing: Review of technology and applications. IEEE Sens J 2012; 12(5): 885-92.

[12] Grattan LS, Meggitt BT, Eds. Optical fiber sensor technology: Advanced applications - Bragg gratings and distributed sensors. Springer 2000.

[13] López-Higuera JM, Ed. Handbook of optical fibre sensing technology. $1^{\text {st }}$ ed. New York: Wiley 2002.

[14] Thévenaz L. Ed. Advanced fiber optics. Lausanne: EPFL Press 2011.

[15] Boyd RW. Nonlinear optics. $3^{\text {rd }}$ ed. Newyork: Academic Press 2008.

[16] Fabelinskii I. Molecular scattering of light. New York: Plenum Press 1968.

[17] Lieberman RA, Mendoza EA, Ferrell DJ, et al. Intrinsic fiber optic sensor for distributed water detection. In: Lieberman RA, Ed. Proceedings of SPIE - The international society for optical engineering. vol. 2068. Boston, MA, USA: Publ by Society of photo-optical instrumentation engineers, Bellingham. WA: USA 1994; pp. 192201.

[18] Kharaz A, Jones B. A distributed optical-fibre sensing system for multi-point humidity measurement. Sensor Actuat A-Phys 1995; 47(1-3): 491-3.

[19] Hadjiloucas S, Keating D, Usher M, et al. Hydrogel based distributed fibre optic sensor for measuring soil salinity and soil water potentials. In: IEE Colloquium (Digest). London: UK 1995; p. 9/1$9 / 6$.

[20] Hadjiloucas S, Keating D, Usher M, Michie W, Culshaw B, McLean A. Hydrogel based distributed fibre optic sensor for measuring moisture content in soils. In: Conference on Lasers and Electro-Optics Europe - Technical Digest; 1996: p. 46.

[21] Michie W, Culshaw B, McLean A, Konstantaki M, Hadjiloucas S. Distributed water ingress and water potential measurements using fibre optics. Cement Concrete Comp 1997; 19(1): 35-44.

[22] MacLean A, Michie W, Pierce S, et al. Hydrogel/fiber optic sensor for distributed measurement of humidity and $\mathrm{pH}$ value. In: Proceedings of SPIE - The International Society for Optical Engineering. vol. 3330. San Diego, CA 1998; pp. 134-44.

[23] Pierce S, MacLean A, Culshaw B. Optical frequency domain reflectometry for interrogation of microbend based optical fibre sensors. In: Proceedings of SPIE - The International Society for Optical Engineering. vol. 3986. Newport Beach, CA, USA: Society of Photo-Optical Instrumentation Engineers, Bellingham. WA: USA 2000; pp. 352-61.

[24] MacLean A, Moran C, Thursby G, Pierce S, Culshaw B, Graham N. Distributed fibre optic sensors for humidity and hydrocarbon detection. In: Proceedings of SPIE - The International Society for Optical Engineering. vol. 3986. Newport Beach, CA, USA: Society of Photo-Optical Instrumentation Engineers, Bellingham. WA: USA $2000 ;$ p. 34251.

[25] Lenke P, Wendt M, Liehr S, Krebber K. Distributed humidity sensing based on Rayleigh scattering in polymer optical fibers. In: Proceedings of SPIE - The international society for optical engineering vol. $7653 ; 2010$.

[26] Falco L, Debergh P, Edye T. Bimorphous distributed transducer for the detection of temperature threshold. In: Anon, Ed. International
Carnahan Conference on Security Technology. Zurich, Switzerland: Publ by IEEE, Piscataway, NJ: USA 1989; 159-62.

[27] Deboux B, Lewis E, Scully P, Edwards R. A novel technique for optical fiber $\mathrm{pH}$ sensing based on methylene blue adsorption. J Lightw Technol 1995; 13(7): 1407-14.

[28] Michie WC, Culshaw B, McKenzie I, et al. Distributed sensor for water and $\mathrm{pH}$ measurements using fiber optics and swellable polymeric systems. Opt Lett 1995; 20(1): 103-5.

[29] Michie W, Culshaw B, Konstantaki M, et al. Distributed pH and water detection using fiber-optic sensors and hydrogels. J Lightw Technol 1995; 13(7): 1415-20.

[30] Buerck J, Sensfelder E, Ache HJ. Distributed sensing of hydrocarbons using evanescent wave interactions in a silicone-clad optical fiber. Proc SPIE - Int Soc Opt Eng 1997; 3105: 21-30.

[31] Chen H, Tu Y, Luo T. A method for oil pipeline leak detection based on distributed fibre optic technology. In: Proceedings of SPIE. Int Soc Opt Eng 1998; 3555: 77-82.

[32] Sensfelder E, Bürck J, Ache HJ. Characterization of a fiber-optic system for the distributed measurement of leakages in tanks and pipelines. Appl Spectrosc 1998; 52(10): 1283-98.

[33] Buerck J, Sensfelder E. Optical fiber sensors for the distributed measurement of hydrocarbons. Proc of SPIE. Int Soc Opt Eng 1999; 3540: 98-109.

[34] MacLean A, Moran C, Johnstone W, Culshaw B, Marsh D, Andrews G. A distributed fibre optic sensor for liquid hydrocarbon detection. In: Udd E ID, editor. Proceedings of SPIE - The International Society for Optical Engineering. vol. 4328. Newport Beach, CA 2001; pp. 47-53.

[35] Buerck J, Roth S, Kraemer K, Mathieu H. OTDR fiber-optical chemical sensor system for detection and location of hydrocarbon leakage. J Hazard Mater 2003; 102(1): 13-28.

[36] King D, Lyons W, Flanagan C, Lewis E. An optical fibre ethanol concentration sensor utilizing fourier transform signal processing analysis and artificial neural network pattern recognition. J Opt A: Pure Appl Opt 2003; 5(4): S69-75.

[37] MacLean A, Moran C, Johnstone W, Culshaw B, Marsh D, Parker P. Detection of solvents using distributed fibre optic sensor. Electron Lett 2003; 39(17): 1237-8.

[38] MacLean A, Moran C, Johnstone W, Culshaw B, Marsh D, Parker $\mathrm{P}$. Detection of hydrocarbon fuel spills using a distributed fibre optic sensor. Sens Actuators, A: Phys 2003; 109(1-2): 60-7.

[39] Cordero SR, Ruiz D, Huang W, Cohen L, Lieberman RA. Intrinsic chemical sensor fibers for extended-length chlorine detection. In: Marcus MA, Culshaw B, Eds. Proceedings of SPIE - The International Society for Optical Engineering. vol. 5589. Philadelphia, PA: 2004; pp. 25-33.

[40] King D, Lyons W, Flanagan C, Lewis E. An optical-fiber sensor for use in water systems utilizing digital signal processing techniques and artificial neural network pattern recognition. IEEE Sens J 2004; 4(1): 21-7.

[41] Cordero S, Beshay M, Low A, Mukamal H, Ruiz D, Lieberman RA. A distributed fiber optic chemical sensor for hydrogen cyanide detection. In: Vo-Dinh T, GG, Lieberman RA, Eds. Proceedings of SPIE - The International Society for Optical Engineering. vol. 5993. Boston, MA 2005.

[42] Mukamal H, Cordero S, Ruiz D, Beshay M, Lieberman R. Distributed fiber optic chemical sensor for hydrogen sulfide and chlorine detection. In: Marcus M A DJ Culshaw B, editor. Proceedings of SPIE - The International Society for Optical Engineering. vol. 6004. Boston, MA 2005.

[43] Sumida S, Okazaki S, Asakura S, Nakagawa H, Murayama H, Hasegawa T. Distributed hydrogen determination with fiber-optic sensor. Sens Actuators B: Chem 2005; 108(1-2): 508-14.

[44] Lee JR, Yoon CY, Dhital D, Yoon DJ. All-fiber optic chemical sensors for public safety monitoring. vol. 123-125. Trans Tech Publications; 2010.

[45] Kinet D, Caucheteur C, Wuilpart M, Megret P. Quasi-distributed measurement of surrounding refractive index using photoncounting time domain reflectometry. In: 2011 IEEE Sens 2011: 355-8.

[46] Okazaki S, Nakagawa H, Murata K, Fukuda K. A distributed hydrogen gas sensor using high refractive index optical fiber. Sens Lett 2011; 9(2): 688-90.

[47] Yun CY, Dhital D, Lee JR, Park G, Kwon IB. Design of multiplexed fiber optic chemical sensing system using clad-removable optical fibers. Opt Laser Technol 2012; 44(1): 269-80. 
[48] Guangping X, Leong Keey S, Asundi A. Optical time-domain reflectometry for distributed sensing of the structural strain and deformation. Opt Lasers Eng 1999; 32(5): 437-47.

[49] Sasaki H, Kubota Y, Watanabe K. Sensitivity property of a heterocore splice fiber optic displacement sensor. In: Proceedings of SPIE - The International Society for Optical Engineering. vol. 5579; 2004. pp. 136-43.

[50] Chai J, Wei S, Chang X, Liu J. Monitoring deformation and damage on rock structures with distributed fiber optical sensing. Int $\mathbf{J}$ Rock Mech Mini Sci 2004; 41(Supplement 1): 298-303.

[51] Aulakh N, Chhabra J, Singh N, Jain S. Microbend resolution enhancing technique for fiber optic based sensing and monitoring of landslides. Exp Tech 2004; 28(3): 37-42.

[52] Aulakh N, Chhabra J, Kamar A, Aggarwal A. Development of a fiber optic based system to monitor landslide activity. IETE Technical Review (Institution of Electronics and Telecommunication Engineers, India). 2004; 21(1):75-81.

[53] Nakamura K, Husdi I, Ueha S. A distributed strain sensor with the memory effect based on the POF OTDR. In: Proceedings of SPIE The International Society for Optical Engineering. vol. 5855 PART II; 2005. p. 807-10.

[54] Lenke P, Krebber K. A new model to calculate the Rayleigh scattering profiles in polymer optical fibers for distributed strain sensing. In: Proceedings of SPIE - The International Society for Optical Engineering. vol. 7503; 2009.

[55] Liehr S, Wendt M, Krebber K. Distributed strain measurement in perfluorinated polymer optical fibres using optical frequency domain reflectometry. Measure Sci Technol 2010; 21(9): 94023.

[56] Liehr S, Nöther N, Krebber K. Incoherent optical frequency domain reflectometry and distributed strain detection in polymer optical fibers. Measure Sci Technol 2010; 21(1).

[57] Zhu ZW, Liu DY, Yuan QY, Liu B, Liu JC. A novel distributed optic fiber transduser for landslides monitoring. Opt Lasers Eng 2011; 49(7): 1019-24

[58] Bravo M, Saenz J, Bravo-Navas M, Lopez-Amo M. Concrete Beam Bending Test Monitorization Using a High Strain Fiber Optic Sensor. J Lightw Technol 2012; 30(8): 1085-9.

[59] Benjamin Varghese P, Vinod P, Jibukumar, Madhusoodanan K, Radhakrishnan P. A quasi distributed fiber optic weightdisplacement sensor using macro bends. Opt Fiber Technol 2012; 18(4): 215-9.

[60] Hille R, Bueker H, Haesing F. Glass fibers as radiation detectors. Nucl Inst Methods Phys Res, A 1990; 299(1-3): 217-21.

[61] Henschel H, Kohn O, Schmidt H, Kirchof J, Unger S. Radiationinduced loss of rare earth doped silica fibres. IEEE T Nucl Sci 1998; 45(3): 1552-7.

[62] Henschel H, Körfer M, Kuhnhenn J, Weinand U, Wulf F. Fibre optic radiation sensor systems for particle accelerators. Nucl Ins Methods Phys Res Sec A 2004; 526(3): 537-50.

[63] Juskaitis R, Mamedov AM, Potapov VT, Shatalin SV. Interferometry with Rayleigh backscattering in a single-mode optical fiber. Opt Lett 1994; 19(3): 225-7.

[64] Park J, Lee W, Taylor H. A fiber optic intrusion sensor with the configuration of an optical time domain reflectometer using coherent interference of Rayleigh backscattering. In: Proceedings of SPIE - The International Society for Optical Engineering. vol. 3555; 1998. pp. 49-56.

[65] Juarez J, Maier E, Choi KN, Taylor H. Distributed fiber-optic intrusion sensor system. J Lightw Technol 2005; 23(6): 2081-7.

[66] Juarez JC, Taylor HF. Polarization discrimination in a phasesensitive optical time-domain reflectometer intrusion-sensor system. Opt Lett 2005; 30(24): 3284-6.

[67] Gorshkov B, Paramonov V, Kurkov A, Kulakov A, Zazirnyi M. Distributed external-action sensor based on a phase-sensitive fibre reflectometer. Quant Electron 2006; 36(10): 963-5.

[68] Madsen C, Bae T, Snider T. Intruder signature analysis from a phase-sensitive distributed fiber-optic perimeter sensor. In: Proceedings of SPIE - The International Society for Optical Engineering. vol. 6770; 2007.

[69] Madsen C, Bae T, Atkins R. Long Fiber-Optic Perimeter Sensor: Signature Analysis. In: Conference on Lasers and ElectroOptics/Quantum Electronics and Laser Science Conference and Photonic Applications Systems Technologies. OSA Technical Digest (CD). Optical Society of America; 2007. p. PWA5.
[70] Juarez J, Taylor H. Field test of a distributed fiber-optic intrusion sensor system for long perimeters. Appl Opt 2007; 46(11): 196871.

[71] Xie K, Rao Y, Ran Z. Distributed optical fiber sensing system based of Rayleigh scattering light $\varphi$-OTDR using single-mode fiber laser with high power and narrow linewidth. Acta Opt Sin 2008; 28(3): 569-72.

[72] Madsen C, Snider T, Atkins R, Sirncik J. Real-time processing of a phase-sensitive distributed fiber optic perimeter sensor. In: Proceedings of SPIE - The International Society for Optical Engineering. vol. 6943; 2008. .

[73] Rao YJ, Luo J, Ran ZL, Yue JF, Luo XD, Zhou Z. Long-distance fiber-optic $\varphi$-OTDR intrusion sensing system. In: Proceedings of SPIE - The International Society for Optical Engineering. vol. 7503; 2009.

[74] Luo J, Rao Y, Yue J, Ran Z. Highly sensitive distributed optical fiber intrusion monitoring system. Chinese J Sci Instrun 2009; 30(6): 1123-8.

[75] Lu Y, Zhu T, Chen L, Bao X. Distributed Vibration Sensor Based on Coherent Detection of Phase-OTDR. J Lightw Technol 2010.

[76] Mullens S, Lees G, Duvivier G. Fiber-optic distributed vibration sensing provides technique for detecting sand production. In: Proceedings of the Annual Offshore Technology Conference. vol. 1. Houston, TX 2010. pp. 432-44.

[77] Qin Z, Zhu T, Chen L, Bao X. High Sensitivity Distributed Vibration Sensor Based on Polarization-Maintaining Configurations of Phase-OTDR. IEEE Photon Technol Lett 2011; 23(15): 109-3.

[78] Pan Z, Liang K, Ye Q, Cai H, Qu R, Fang Z. Phase-sensitive OTDR system based on digital coherent detection. In: Proceedings of SPIE - The International Society for Optical Engineering. vol. 8311; 2011.

[79] Lu Y, Zhu T, Bao X, Chen L. Vibration monitoring with high frequency response based on coherent phase-sensitive OTDR method In: Proceedings of SPIE - The International Society for Optical Engineering. vol. 7753. Ottawa, ON; 2011.

[80] Zhou DP, Qin Z, Li W, Chen L, Bao X. Distributed vibration sensing with time-resolved optical frequency-domain reflectometry. Opt Express 2012; 20(12): 13138-45.

[81] Qin Z, Chen L, Bao X. Continuous wavelet transform for nonstationary vibration detection with phase-OTDR. Opt Express 2012; 20(18): 20459-65.

[82] Owen A, Duckworth G, Worsley J. OptaSense: Fibre Optic Distributed Acoustic Sensing for Border Monitoring. In: Intelligence and Security Informatics Conference (EISIC), 2012 European; 2012. pp. $362-64$.

[83] Liang K, Pan Z, Zhou J, Ye Q, Cai H, Qu R. Multi-parameter vibration detection system based on phase sensitive optical time domain reflectometer. Chin J Lasers 2012; 39(8).

[84] Ding Z, Yao XS, Liu T, et al. Long-range vibration sensor based on correlation analysis of optical frequency-domain reflectometry signals. Opt Express 2012; 20(27): 28319-29.

[85] Froggatt M, Moore J. High-Spatial-Resolution Distributed Strain Measurement in Optical Fiber with Rayleigh Scatter. Appl Opt 1998; 37(10): 1735-40.

[86] Rogers AJ. Polarisation optical time domain reflectometry. Electron Lett 1980; 16(13): 489-90.

[87] Strutt J. XXXVI. On the light from the sky, its polarization and colour. Philosophical Mag Ser 1871; 41(273): 274-9.

[88] Kapron FP, Maurer RD, Teter MP. Theory of Backscattering Effects in Waveguides. Appl Opt 1972; 11(6): 1352-6.

[89] Brinkmeyer E. Analysis of the backscattering method for singlemode optical fibers. J Opt Soc Am 1980; 70(8): 1010-2.

[90] Nakazawa M. Rayleigh backscattering theory for single-mode optical fibers. J Opt Soc Am 1983; 73(9): 1175-80.

[91] Nakazawa M. Theory of backward Rayleigh scattering in polarization-maintaining single-mode fibers and its application to polarization optical time domain reflectometry. IEEE J Quant Electron 1983; 19: 854-61.

[92] Hartog A, Gold M. On the theory of backscattering in single-mode optical fibers. J Lightw Technol 1984; 2(2): 76-2.

[93] Senior JM. Optical fiber communications (2nd ed.): principles and practice. Hertfordshire, UK, UK: Prentice Hall International (UK) Ltd. 1992.

[94] Born M, Wolf E, Bhatia A. Principles of Optics: Electromagnetic Theory of Propagation, Interference and Diffraction of Light. Cambridge University Press 1999. 
[95] Young AT. Rayleigh scattering. Appl Opt 1981; 20(4): 533-5.

[96] Rogers AJ, Handerek VA. Frequency-derived distributed opticalfiber sensing: Rayleigh backscatter analysis. Appl Opt 1992; 31(21): 4091-5.

[97] Gysel P, Staubli R. Statistical properties of Rayleigh backscattering in single-mode fibers. J Lightw Technol 1990; 8(4): 561-7.

[98] Barnoski MK, Jensen SM. Fiber waveguides: a novel technique for investigating attenuation characteristics. Appl Opt 1976; 15: 21125 .

[99] Hartog A, Leach A, Gold M. Distributed temperature sensing in solid-core fibres. Electron Lett 1985; 21(23): 1061-2.

[100] Zoboli M, Bassi P. High spatial resolution OTDR attenuation measurements by a correlation technique. Appl Opt 1983; 22(23): $3680-1$.

[101] Bernard JJ, Depresles E. High-Resolution Correlation Otdr for Distributed Fiber-Optic Sensors and Mobile Cabling. Fiber Integrated Opt 1988:7(2):79-84

[102] Nazarathy M, Newton S, Giffard R, et al. Real-time long range complementary correlation optical time domain reflectometer. J Lightw Technol 1989;7(1):24-38

[103] Jones M. Using simplex codes to improve OTDR sensitivity. IEEE Photon Technol Lett 1993;5(7):822-24.

[104] Lee D, Yoon H, Kim NY, Lee H, Park N. Analysis and experimental demonstration of simplex coding technique for SNR enhancement of OTDR. In: Proceedings of the Lightwave Technologies in Instrumentation and Measurement Conference 2004;118-22.

[105] Lee D, Yoon H, Kim P, Park J, Kim NY, Park N. SNR enhancement of OTDR using biorthogonal codes and generalized inverses. IEEE Photon Technol Lett 2005;17(1):163-65.

[106] Levine B, Bethea C, Cohen L, Campbell J, Morris G. Optical time domain reflectometer using a photon-counting InGaAs/InP avalanche photodiode at $1.3 \mu \mathrm{m}$. Electron Lett 1985; 21(2): 83-84.

[107] Bethea CG, Levine BF, Cova S, Ripamonti G. High-resolution and high-sensitivity optical-time-domain reflectometer. Opt Lett 1988; 13(3): 233-35.

[108] Eraerds P, Legré M, Zhang J, Zbinden H, Gisin N. Photon Counting OTDR: Advantages and Limitations. J Lightw Technol 2010; 28(6): 952-64.

[109] Healey P. Fading in heterodyne OTDR. Electron Lett 1984; 20(1): 30-32.

[110] Shimizu K, Horiguchi T, Koyamada Y. Characteristics and reduction of coherent fading noise in Rayleigh backscattering measurement for optical fibers and components. J Lightw Technol 1992; 10(7): 982-87.

[111] Bodtker E, Tromborg B, Mark J, Nielsen C. Heterodyne OTDR at $0.82 \mu \mathrm{m}$. Electron Lett 1983; 19(10): 361

[112] King J, Smith D, Richards K, Timson P, Epworth R, Wright S. Development of a coherent OTDR instrument. J Lightw Technol 1987; 5(4): 616-24.

[113] Eickhoff W, Ulrich R. Optical frequency domain reflectometry in single-mode fiber. Appl Phys Lett 1981; 39(9): 693-95.

[114] Yuksel K, Wuilpart M, Moeyaert V, Mégret P. Optical frequency domain reflectom etry: A review. In: 11th International Conference on Transparent Optical Networks, 2009. ICTON '09; 2009; 1-5.

[115] Fan X, Koshikiya Y, Ito F. Phase-Noise-Compensated Optical Frequency-Domain Reflectometry. IEEE J Quant Electron 2009; 45(6): 594-602.

[116] Ito F, Fan X, Koshikiya Y. Long-Range Coherent OFDR With Light Source Phase Noise Compensation. J Lightw Technol 2012;30(8):1015-24.

[117] Someda CG. Electromagnetic Waves. CRC Press 2006

[118] Wanser KH, Voss KF. Crack detection using multimode fiber optical time domain reflectometry. In: Kersey Alan D DJP, editor. Proceedings of SPIE - The International Society for Optical Engineering. vol. 2294. San Diego, CA, USA: Society of Photo-Optical Instrumentation Engineers, Bellingham, WA, United States 1994; 43-52.

[119] Liu H, Yang Z. Distributed optical fiber sensing of cracks in concrete. In: Proceedings of SPIE - Int Soc Opt Eng1998; (3555) 29199.

[120] Pierce SG, MacLean A, Culshaw B. Optical frequency-domain reflectometry for microbend sensor demodulation. Appl Opt 2000;39(25):4569-81.
[121] Wei B, Wu C, Du Y. Research on optical fiber strain sensor based on the spiral bending. In: Proceedings of SPIE - Int Soc Opt Eng 2005(5633) 171-6.

[122] Binu S, Pillai V, Chandrasekaran N. OTDR based fiber optic microbend sensor for distributed sensing applications in structural pressure monitoring. J Opt 2006;35(1):36-44.

[123] Wan K, Leung C. Applications of a distributed fiber optic crack sensor for concrete structures. Sens Actuators, A: Phys 2007; 135(2): 458-64.

[124] Tang TG, Chen CH, Liu HW. Application of distributed optical fiber sensors into crack monitoring of dam's foundation. Chin J Sens Actuat 2007; 20(10):2357-60.

[125] Boiarski A, McGinniss V, Turhan-Sayan G. High spatial resolution distributed fiber optic temperature sensor. In: Proceedings of SPIE - Int Soc Opt Eng1990; (1338)18-30.

[126] Boiarski A, Pilate G, Fink T, Nilsson N. Temperature measurements in power plant equipment using distributed fiber optic sensing. IEEE Transactions Power Deliv 1995; 10(4): 1771 -78.

[127] Husdi I, Nakamura K, Ueha S. Sensing characteristics of plastic optical fibres measured by optical time-domain reflectometry. Measure Sci Technol 2004;15(8):1553-59.

[128] Hartog A. A distributed temperature sensor based on liquid-core optical fibers. J Lightw Technol1983;1(3):498 -509.

[129] Farries M, Fermann M, Laming R, Poole S, Payne D, Leach A. Distributed temperature sensor using $\mathrm{Nd}^{3+}$-doped optical fibre. Electron Lett 1986; 22(8):418 -9.

[130] Ko PKY, Demokan S, Tam H. Distributed temperature sensing with erbium-doped fiber amplifiers. J Lightw Technol 1996; 14(10): $2236-45$.

[131] Juskaitis R, Mamedov AM, Potapov VT, Shatalin SV. Distributed interferometric fiber sensor system. Opt Lett 1992;17(22):1623-25.

[132] Rathod R, Pechstedt RD, Jackson DA, Webb DJ. Distributed temperature-change sensor based on Rayleigh backscattering in an optical fiber. Opt Lett1994; 19(8): 593-5.

[133] Lopatin C, Froggatt M, Childers B, Rogowski R. Distributed measurement of strain in smart materials using Rayleigh scattering. In: International SAMPE Technical Conference 2000: (32): 231-41.

[134] Gifford D, Soller B, Wolfe M, Froggatt M. Distributed fiber-optic temperature sensing using Rayleigh backscatter. In: 31st European Conference on Optical Communication, 2005. ECOC 2005; (3) 511-2.

[135] Klute S, Duncan R, Fielder R, Butler G, Mabe J, Sang A, et al. Fiber-optic shape sensing and distributed strain measurements on a morphing chevron. In: Collection of Technical Papers - 44th AIAA Aerospace Sciences Meeting. Reno 2006(10):7460-82.

[136] Soller B, Gifford D, Wolfe M, Froggatt M, Yu M, Wysocki P. Measurement of localized heating in fiber optic components with millimeter spatial resolution. In: 2006 Optical Fiber Communication Conference, and the 2006 National Fiber Optic Engineers Conference. vol. 2006; 2006

[137] Duncan R, Froggatt M, Kreger S, et al. High-accuracy fiber-optic shape sensing. In: Proceedings of SPIE - Int Soc Opt Eng 2007p.(6530).

[138] Gifford D, Kreger S, Sang A, Froggatt M, Duncan R, Wolfe M, et al. Swept-wavelength interferometric interrogation of fiber Rayleigh scatter for distributed sensing applications. In: Proceedings of SPIE - Int Soc Opt Eng 2007; p. (6770).

[139] Kreger S, Gifford D, Froggatt M, Sang A, Duncan R, Wolfe M, et al. High-resolution extended distance distributed fiber-optic sensing using Rayleigh backscatter. In: Proceedings of SPIE - Int Soc Opt Eng 2007; p.(6530).

[140] Sang A, Froggatt M, Gifford D, Kreger S, Dickerson B. One Centimeter Spatial Resolution Temperature Measurements in a Nuclear Reactor Using Rayleigh Scatter in Optical Fiber. IEEE Sens J 2008; 8(7):1375 -80.

[141] Kreger S, Sang A, Gifford D, Froggatt M. Distributed strain and temperature sensing in plastic optical fiber using Rayleigh scatter. In: Proceedings of SPIE - Int Soc Opt Eng 2009; p. (7316).

[142] Lanticq V, Bourgeois E, Magnien P, Dieleman L, Vinceslas G, Sang A, et al. Soil-embedded optical fiber sensing cable interrogated by Brillouin optical time-domain reflectometry (B-OTDR) and optical frequency-domain reflectometry (OFDR) for embedded cavity detection and sinkhole warning system. Measure Sci Technol 2009; 20(3): 034018. 
[143] Maier R, MacPherson W, Barton J. Distributed transverse load sensing in polarisation maintaining fibres using Rayleigh backscatter. In: Proceedings of SPIE - Int Soc Opt Eng 2009;(7503).

[144] Gifford D, Sang A, Kreger S, Froggatt M. Strain measurements of a fiber loop rosette using high spatial esolution Rayleigh Scatter distributed sensing. In: Proceedings of SPIE - Int Soc Opt Eng 2010;( 7653).

[145] Maier RRJ, MacPherson WN, Barton JS, McCulloch S, Jones BJS. Distributed sensing using Rayleigh scatter in polarizationmaintaining fibres for transverse load sensing. Measure Sci Technol 2010; 21(9): 094019.

[146] Henault J, Salin J, Moreau G, et al. Monitoring of concrete structures using OFDR technique. In: AIP Conference Proceedings. San Diego, CA; 2011;(1335)1386-93.

[147] Sanborn E, Sang A, Wesson E, Wigent III D, Lucier G. Distributed fiber optic strain measurement using rayleigh scatter in composite structures. In: Conference Proceedings of the Society for Experimental Mechanics Series 2011: (6)461-70.

[148] Sang A, Froggatt M, Kreger S, Gifford D. Millimeter resolution distributed dynamic strain measurements using optical frequency domain reflectometry. In: Proceedings of SPIE - The International Society for Optical Engineering. Ottawa, ON; 2011(7753).

[149] Boyd C, Lally E, Horrell E, Dickerson B. Verifying cryogenic cooling of superconducting cables using optical fiber. In: Future of Instrumentation International Workshop (FIIW), 2012; 2012:1-4.

[150] Chen T, Wang Q, Chen R, Zhang B, Chen K. Active Distributed Sensing using Self-heated Optical Fibers. In: CLEO: Science and Innovations. OSA Technical Digest (online). Opt Soc Am 2012: CM4B.2.

[151] Chen T, Wang Q, Chen R, et al. Distributed hydrogen sensing using in-fiber Rayleigh scattering. Appl Phys Lett 2012; 100(19): 191105-3.

[152] Chen T, Wang Q, Chen R, et al. Distributed high-temperature pressure sensing using air-hole microstructural fibers. Opt Lett 2012; 37(6): 1064-6.

[153] Chen T, Wang Q, Chen R, Zhang B, Lin Y, Chen KP. Distributed liquid level sensors using self-heated optical fibers for cryogenic liquid management. Appl Opt 2012; 51(26): 6282-9.

[154] Faustov A, Gusarov A, Wuilpart M, et al. Distributed optical fibre temperature measurements in a low dose rate radiation environment based on Rayleigh backscattering. In: Proceedings of SPIE - The International Society for Optical Engineering 2012; 8439.

[155] Gifford D, Froggatt M, Sang A, Kreger S. Multiple Fiber Loop Strain Rosettes in a Single Fiber Using High Resolution Distributed Sensing. IEEE Sens J 2012; 12(1): 55-63.

[156] Henault JM, Quiertant M, Delepine-Lesoille S, et al. Quantitative strain measurement and crack detection in RC structures using a truly distributed fiber optic sensing system. Constr Build Mater 2012; 37: 916-23.

[157] Lally E, Reaves M, Horrell E, Klute S, Froggatt M. Fiber optic shape sensing for monitoring of flexible structures. In: Proceedings of SPIE - The International Society for Optical Engineering 2012; 8345.

[158] Villalba S, Casas JR. Application of optical fiber distributed sensing to health monitoring of concrete structures. Mech Syst Signal Pr 2012. 39(1-2): 441-51

[159] Froggat ME. Rayleigh Backscattering Signatures of Optical Fibers: Their Properties and Applications. In: OFC 2013. Anahaim, USCA 2013.

[160] Rogers AJ, Handerek VA. High-resolution frequency-derived distributed optical fiber sensing. In: Kersey Alan D DJP, Ed. Proceedings of SPIE - The International Society for Optical Engineering. San Diego, CA, USA: Society of Photo-Optical Instrumentation Engineers, Bellingham, WA, United States 1994; 2294: p. 2-13.

[161] Mamedov A, Potapov V, Shatalin S, Juskaitis R. The use of Rayleigh backscattering for interferometric remote fiber optic sensor system. Turk J Phys 1996; 20(4):340-3.

[162] Potapov V, Treshchikov V, Shatalin S. Interference Phenomena Induced by the Rayleigh Scattering in Optical Fibers. J Commun Technol El 1998; 43(12): 1407-11.

[163] Shatalin SV, Treschikov VN, Rogers AJ. Interferometric Optical Time-Domain Reflectometry for Distributed Optical-Fiber Sensing. Appl Opt 1998; 37(24): 5600-4.

[164] Posey JR, Johnson G, Vohra S. Strain sensing based on coherent Rayleigh scattering in an optical fibre. Electron Lett 2000; 36(20): 1688-9.
[165] Koyamada Y, Eda Y, Hirose S, Nakamuka S, Hogari K. Novel fiber-optic distributed strain and temperature sensor with very high resolution. IEICE Trans Commun 2006; E89-B(5):1722-5.

[166] Imahama M, Koyamada Y, Hogari K. Restorability of Rayleigh backscatter traces measured by coherent OTDR with precisely frequency-controlled light source. IEICE Trans Commun 2008; E91B(4): 1243-6.

[167] Koyamada Y, Imahama M, Kubota K, Hogari K. Fiber-Optic Distributed Strain and Temperature Sensing With Very High Measurand Resolution Over Long Range Using Coherent OTDR. J Lightw Technol 2009 May; 27(9):1142-6.

[168] Zhi Y, Pengxiang S, Yongqian L. Research on COTDR for measuring distributed temperature and strain. In: 2011 Second International Conference on Mechanic Automation and Control Engineering (MACE) 2011: pp. 590-3.

[169] Kung A, Budin J, Thevenaz L, Robert P. Rayleigh fiber optics gyroscope. IEEE Photon Technol Lett 1997; 9(7): 973-5.

[170] Yuan L, Liu Z, Yang J. Rayleigh backscattering fiber optic rotation sensor based on combined two-ring-resonator. Sens Actuators APhys 2007; 136(1): 216-20.

[171] Yanlei L, Haili J, Libo Y. Multi-ring compound fiber optic rotation measuring approach based on the Rayleigh Backscattering. In: 2012 International Conference on Measurement, Information and Control (MIC) 2012; 1: p. 107-12.

[172] Shiota T, Hidaka H, Suzuki F, Fukuda O, Inada K. Temperature Distribution Sensor Using Silica Based Optical Fiber. In: 11th European Conference on Optical Communication Venice, Italy 1985; 1: p. 805-8.

[173] Kingsley SA, McGinniss VD. DISTRIBUTED FIBER-OPTIC HOT-SPOT SENSORS. In: De Paula Ramon P UE, editor. Proceedings of SPIE - The International Society for Optical Engineering, Cambridge, MA, Engl: SPIE, Bellingham, WA, USA 1986; 718: p. 218-24.

[174] Bock WJ, Domanski AW, Wolinski TR. Pressure and temperature effects on beat length in highly birefringent optical fibers. In: Proceedings of SPIE - The International Society for Optical Engineering 1990; 1267: p. 50-5.

[175] Boiarski A. Distributed fiber optic temperature sensing. In: Applications of Fiber Optic Sensors in Engineering Mechanics 1993; p. 210-24.

[176] Hypszer R, Kosmowski B, Pluciński J, Wierzba P. Fiber optic quasi-distributed temperature sensor. In: Proceedings of SPIE - The International Society for Optical Engineering 1996; 3054: p. 122-5.

[177] Julian PL, Farhadiroushan M, Handerek VA, Rogers AJ. High spatial resolution distributed optical-fibre strain or temperature sensing. Proceedings of SPIE - The International Society for Optical Engineering 1999; 3541: 297-309.

[178] Rogers A, Wuilpart M, Blondel M. New polarimetry for fullydistributed optical-fibre strain and temperature sensing. In: Proceedings of SPIE - The International Society for Optical Engineering. Newport Beach, CA, USA: Society of Photo-Optical Instrumentation Engineers, Bellingham, WA, United States 2000; 3986: pp. 302-11.

[179] Donlagic D, Lesic M. All-fiber quasi-distributed polarimetric temperature sensor. Opt Expr 2006;14(22):10245-54.

[180] Crunelle C, Legré M, Wuilpart M, Mégret P, Gisin N. Distributed temperature sensor interrogator based on polarization-sensitive reflectometry. IEEE Sens J 2009; 9(9): 1125-9.

[181] Cogdell G. Fiber optic sensors for intruder detection. In: Institute of Electrical and Electronics Engineers 1988 International Carnahan Conference on Security Technology, 1988. Crime Countermeasures, Proceedings 1988: p. 19-23.

[182] Ulrich R, Simon A. Polarization optics of twisted single-mode fibers. Appl Opt 1979;18: 2241-51.

[183] Rashleigh SC, Ulrich R. High birefringence in tension-coiled single-mode fibers. Opt Lett 1980; 5(8): 354-6.

[184] Ulrich R, Rashleigh SC, Eickhoff W. Bending-induced birefringence in single-mode fibers. Opt Lett 1980; 5(6): 273-5

[185] Rogers AJ. Polarization-optical time domain reflectometry: a technique for the measurement of field distributions. Appl Opt 1981; 20:1060-74.

[186] Barnoski MK, Rourke MD, Jensen SM, Melville RT. Optical time domain reflectometer. Appl Opt 1977; 16(9): 2375-9.

[187] Farries M, Rogers A. Distributed Sensing Using Stimulated Raman Interaction In A Monomode Optical Fibre. In: Proceedings of SPIE 
- The International Society for Optical Engineering 1984; 514: pp. 121-32.

[188] Dakin J, Pratt D, Bibby G, Ross J. Distributed optical fibre Raman temperature sensor using a semiconductor light source and detector. Electron Lett 1985; 21(13): 569-70.

[189] The Plessey Company Plc, assignee. Temperature measuring arrangement. GB2140554 (A) 1984.

[190] Horiguchi T, Kurashima T, Tateda M. Tensile strain dependence of Brillouin frequency shift in silica optical fibers. IEEE Photon Technol Lett 1989; 1(5): 107-8.

[191] Culverhouse D, Farahi F, Pannell C, Jackson D. Potential of stimulated Brillouin scattering as sensing mechanism for distributed temperature sensors. Electron Lett1989; 25(14): 913-5.

[192] Ross JN. Measurement of magnetic field by polarisation optical time-domain reflectometry. Electron Lett 1981;17(17): 596-7.

[193] Kim BY, Choi SS. Analysis and measurement of birefringence in single-mode fiber using the backscattering method. Opt Lett 1981; 6: 578-80.

[194] Kim BY, Park D, Choi SS. Use of polarization-optical time domain reflectometry for observation of the Faraday effect in single-mode fibers. IEEE J Quantum Electron 1982; 18(4): 455-6.

[195] Galtarossa A, Menyuk CR. Polarization Mode Dispersion. New York: Springer 2005.

[196] Corsi F, Galtarossa A, Palmieri L. Polarization mode dispersion characterization of single-mode optical fiber using backscattering technique. J Lightwave Technol 1998; 16(10):1832-43.

[197] Sunnerud H, Olsson BE, Andrekson PA. Technique for characterization of polarisation mode dispersion accumulation along optical fibres. Electron Lett 1998; 34: 397-8.

[198] Huttner B, Gisin B, Gisin N. Distributed PMD measurement with a polarization-OTDR in optical fibers. J Lightw Technol 1999;17: $1843-7$.

[199] Wuilpart M, Rogers A, Megret P, Blondel M. Distributed measurement of the polarization properties of a fibre using a backscatter technique. In: Proceedings of SPIE - The International Society for Optical Engineering. Glasgow, UK: Society of Photo-Optical Instrumentation Engineers, Bellingham, WA, United States 2000; 4074: p. 322-30.

[200] Wuilpart M, Ravet G, Megret P, Blondel M. Polarization mode dispersion mapping in optical fibers with a polarization-OTDR. IEEE Photon Technol Lett 2002; 14(12): 1716-8.

[201] Galtarossa A, Palmieri L. Spatially resolved PMD measurements. J Lightw Technol 2004; 22(4):1103-15.

[202] Dong H, Shum P, Zhou JQ, Ning GX, Gong YD, Wu CQ. Spectralresolved backreflection measurement of polarization mode dispersion in optical fibers. Opt Lett 2007; 32: 1665-67.

[203] Galtarossa A, Grosso D, Palmieri L, Schenato L. Distributed polarization-mode-dispersion measurement in fiber links by polarization-sensitive reflectometric techniques. IEEE Photon Technol Lett 2008; 20(23): 1944-46

[204] Fan X, Koshikiya Y, Ito F. Full polarimetric phase-noisecompensated optical-frequency-domain reflectometry for distributed measurement of high-PMD fibers. Opt Lett 2010;35(1):25-27.

[205] Palmieri L, Fosuhene S, Leitch A, Galtarossa A. Single-end measurement of root mean square differential group delay in singlemode fibers by polarization optical time-domain reflectometry. IEEE Photon Technol Lett 2011; 23(4):260-62.

[206] Rogers AJ. Distributed measurement of strain using optical-fibre backscatter polarimetry. Strain 2000; 36(3):135-42.

[207] Rogers A, Shatalin S, Kanellopoulos S. Distributed measurement using optical-fibre backscatter polarimetry: Proceedings of SPIE The international society for optical engineering 2004; (5502)46367.

[208] Fu S, Wu C, Li Y, Dong X. Signal process in distributed optical fiber sensing system based on P-OTDR: Proceedings of SPIE - The international society for optical engineering 2005;(5634)241-46.

[209] Rogers A, Shatalin S, Kanellopoulos S. Optical-fibre backscatter polarimetry for the distributed measurement of full strain fields: Proceedings of SPIE - The international society for optical engineering 2005; (6004).

[210] Rogers A, Shatalin S, Kanellopoulos S. Distributed measurement of fluid pressure via optical-fibre backscatter polarimetry: Proceedings of SPIE - The international society for optical engineering: 2005;(5855)230-33.

[211] Galtarossa A, Grosso D, Palmieri L, Schenato L. Location and temporal characterization of hinges in optical fiber links: European conference of optical communication;2007;33rd Berlin (DE)10304.

[212] Galtarossa A, Grosso D, Palmieri L, Schenato L. Reflectometric characterization of hinges in optical fiber links. IEEE Photon Technol Lett 2008; 20(10): 854-56.

[213] Galtarossa A, Grosso D, Palmieri L, Schenato L. Reflectometric characterization of hinges in fiber-optic links: Optical fiber communications conference; 2008; Optical Society of America; San Diego (US-CA)

[214] Zhang Z, Bao X. Distributed optical fiber vibration sensor based on spectrum analysis of Polarization-OTDR system. Opt Exp 2008;16(14):10240-47.

[215] Zhang Z, Bao X. Continuous and damped vibration detection based on fiber diversity detection sensor by rayleigh backscattering. J Lightwave Technol 2008; 26(7): 832 -38.

[216] Galtarossa A, Grosso D, Palmieri L. Accurate characterization of twist-induced optical activity in single-mode fibers by means of polarization-sensitive reflectometry. IEEE Photon Technol Lett 2009; 21(22):1713-15

[217] Galtarossa A, Grosso D, Palmieri L. Reflectometric measurement of strain induced optical activity coefficient in single-mode randomly birefringent twisted fibers: European conference on optical communications; IEEE 2009;35th, Vienna (A)1-2.

[218] Palmieri L. Accurate distributed characterization of polarization properties in optical fibers: European conference on optical communications; IEEE 2010; 36th, Torino (IT)1-6.

[219] Rogers A. A wavelength-diversity method for complete spatial polarization profiling in optical fibers. J Lightw Technol 2011; 29(15):2223-28.

[220] Palmieri L, Galtarossa A. Reflectometric fiber optic sensor for distributed measurement of intense magneto-static fields: IEEE; 2011; Sensors, Limerick (IR) 117-20.

[221] Palmieri L, Galtarossa A. Distributed polarization-sensitive reflectometry in nonreciprocal single-mode optical fibers. J Lightw Technol 2011; 29(21): 3178-84.

[222] Wuilpart M, Caucheteur C, Goussarov A, Aerssens M, Massaut V, Megret P. Measurement of magnetic field using Rayleigh backscattering in optical fibres :2nd international conference on advancements in nuclear instrumentation measurement methods and their applications 2011 (ANIMMA) $1-6$.

[223] Aerssens M, Gusarov A, Moreau P, et al. Development of a jones vector based model for the measurement of a plasma current in a thermonuclear fusion reactor with a POTDR setup: Proceedings of SPIE - The international society for optical engineering;2012(8439).

[224] Palmieri L, Galtarossa A. Distributed fiber optic sensor for mapping of intense magnetic fields based on polarization sensitive reflectometry: Proceedings of SPIE;2012; Sydney (8351).

[225] Collett E. Polarized light, fundamentals and applications. New York: Dekker 1993.

[226] Galtarossa A, Grosso D, Palmieri L, Rizzo M. Spin-profile characterization in randomly birefringent spun fibers by means of frequency-domain reflectometry. Opt Lett 2009; 34(7):1078-80.

[227] Palmieri L, Galtarossa A, Geisler T. Distributed characterization of bending effects on the birefringence of single-mode optical fibers. Opt Lett 2010; 35(14): 2481-83.

[228] Palmieri L, Geisler T, Galtarossa A. Limits of applicability of polarization sensitive reflectometry. Opt Exp 2011;19(11):1087479 .

[229] Jones RC. A new calculus for the treatment of optical systems. Part VI. J Opt Soc Am 1947; 37:110-2.

[230] Faraday M. On the magnetic affection of light, and on the distinction between the ferromagnetic and diamagnetic conditions of matter. Philosoph Mag Series 3. 1846; 29(193):153-56.

[231] Noda J, Hosaka T, Sasaki Y, Ulrich R. Dispersion of Verdet constant in stress-birefringent silica fibre. Electron Lett 1984; 20(22): 906-08.

[232] Cruz JL, Andres MV, Hernandez MA. Faraday effect in standard optical fibers: dispersion of the effective Verdet constant. Appl Opt 1996; 35(6): 922-27.

[233] Kanatani K. Analysis of 3-D rotation fitting. IEEE Trans Pattern Anal Mach Intell 1994; 16(5): 543-49.

[234] Jopson R, Nelson L, Kogelnik H. Measurement of second-order polarization-mode dispersion vectors in optical fibers. IEEE Photon Technol Lett 1999;11(9):1153 -55. 
[235] Ross JN. The rotation of the polarization in low birefringence monomode optical fibres due to geometric effects. Opt Quant Electron 1984;16(5):455-61.

[236] Tomita A, Chiao RY. Observation of berry's topological phase by use of an optical fiber. Phys Rev Lett 1986; 57(8):937-40.
[237] Berry MV. Interpreting the anholonomy of coiled light. Nature 1987; 326(6110): 277-78.

[238] Berry MV. Quantal Phase Factors Accompanying Adiabatic Changes: Proceedings of the Royal Society of London Series A, Mathematical and Physical Sciences; 1984; 392(1802): 45-57.

Received: March 25, 2013

(c) Palmieri and Schenato; Licensee Bentham Open.

This is an open access article licensed under the terms of the Creative Commons Attribution Non-Commercial License (http://creativecommons.org/licenses/by-nc/3.0/) which permits unrestricted, non-commercial use, distribution and reproduction in any medium, provided the work is properly cited. 